\title{
Demand Reduction and Inefficiency in Multi-Unit Auctions
}

\author{
Lawrence M. Ausubel, Peter Cramton, Marek Pycia, Marzena Rostek, and Marek Weretka*
}

March 2014

\begin{abstract}
Auctions often involve the sale of many related goods: Treasury, spectrum and electricity auctions are examples. In multi-unit auctions, a bid for one unit may affect payments for other units won, giving rise to an incentive to shade bids differently across units. We establish that such differential bid shading results generically in ex post inefficient allocations in the uniform-price and pay-as-bid auctions. We also show that, in general, the efficiency and revenue rankings for the two formats are ambiguous. However, in settings with symmetric bidders, the pay-as-bid auction often outperforms. In particular, with diminishing marginal utility, symmetric information and linearity, it yields greater expected revenues. We explain the rankings through multi-unit effects, which have no counterparts in auctions with unit demands. We attribute the new incentives separately to multi-unit but constant marginal utility and diminishing marginal utility.
\end{abstract}

JEL CLASSIFICATiON: D44, D82, D47, L13, L94

Keywords: Multi-Unit Auctions, Demand Reduction, Treasury Auctions, Electricity Auctions

${ }^{*}$ Lawrence M. Ausubel and Peter Cramton: University of Maryland, Department of Economics, College Park, MD 20742; ausubel@econ.umd.edu, pcramton@gmail.com. Marek Pycia: UCLA, Department of Economics, 8283 Bunche Hall, CA 90095; pycia@ucla.edu. Marzena Rostek and Marek Weretka: University of Wisconsin-Madison, Department of Economics, 1180 Observatory Drive, Madison, WI 53706, U.S.A.; rostek@wisc.edu, weretka@wisc.edu. This paper combines the results from "Demand Reduction and Inefficiency in Multi-Unit Auctions" by L. M. Ausubel and P. Cramton and "Design of Divisible Good Markets" by M. Pycia, M. Rostek and M. Weretka. We would like to thank Jen-Wen Chang, Steven Durlauf, Manolis Galenianos, Edward Green, Ali Hortaçsu, Jakub Kastl, Paul Klemperer, Vijay Krishna, Daniel Marszalec, Preston McAfee, Meg Meyer, Stephen Morris, James Wang, Kyle Woodward, Jaime Zender, anonymous referees, and participants at numerous conferences and seminars and for helpful comments and suggestions. Ausubel and Cramton (SES-09-24773 and earlier grants) and Rostek and Weretka (SES-08-51876) gratefully acknowledge the financial support of the National Science Foundation. 


\section{Introduction}

Many markets sell goods or assets to bidders who demand multiple units. Important examples include auctions of government debt, electricity, spectrum, emission permits, and refinancing (repos). One of the preeminent justifications for auctioning public resources is to attain allocative efficiency. Vice President Al Gore opened the December 1994 Broadband PCS spectrum auction proclaiming; "Now we're using the auctions to put licenses in the hands of those who value them the most." ${ }^{1}$ For single-item auctions, the theory, which has built on the seminal efficiency and revenue equivalence results by Vickrey (1961) and the revenue rankings by Milgrom and Weber (1982), informs design and policy. To assess the relative merits of the multi-unit auction formats, none of the classic results can be invoked.

In this paper, we show that the classic conclusions about efficiency and revenue rankings and the possibility of surplus extraction from auctions with unit demands do not hold in multi-unit settings. The key to why the analogy between single-unit and multi-unit auctions does not apply is differential bid shading, the incentive to shade bids differently across units in multi-unit auctions. Such shading arises because a bid for one unit may affect the payments for other units won and, thus, it is enhanced by bidders' market power, observed in many markets. Indeed, even in Treasury auctions, where the number of participants is large, the top five bidders typically purchase nearly one-half of the issue (Malvey and Archibald (1998)). Electricity and spectrum markets exhibit even higher levels of concentration. Demand reduction (supply reduction in the case of electricity auctions) is of great practical importance, both in terms of auction design and participants' bidding strategies and, indeed, has influenced design choices in major markets (Section 6 recalls some prominent cases).

In the majority of multi-unit auctions that are known to us, variants of two formats are used in practice: the pay-as-bid ("discriminatory-price") auction, the traditional format in the U.S. Treasury auctions; and the uniform-price auction, proposed by Milton Friedman (1960) and currently used by the Treasury. In both formats, bidders each submit bids for various quantities at various prices, the auctioneer determines the market-clearing price and accepts all bids exceeding market-clearing price. The two auctions differ in terms of payment: In the pay-as-bid auction, bidders pay their actual bids. In the uniform-price auction, bidders pay the market-clearing price for all units won. ${ }^{2}$ This paper compares these two commonly

\footnotetext{
${ }^{1}$ In the Omnibus Budget Reconciliation Act of 1993, which authorized spectrum auctions, the U.S. Congress established the "efficient and intensive use of the electromagnetic spectrum" as a primary objective of U.S spectrum auctions (47 U.S.C. $\S 309(\mathrm{j})(3)(\mathrm{D}))$.

${ }^{2}$ The cross-country study on Treasury practices by Brenner, Galai and Sade (2009) reports that, out of the 48 countries surveyed, 24 use a pay-as-bid auction to finance public debt, 9 use a uniform-price auction, and 9 employ both auction formats, depending on the type of security being issued; the remaining 6 use a different mechanism. In the United States, the Treasury has been using the pay-as-bid auction to sell Treasury bills since 1929 and to issue notes and bonds since the 1970s. In November 1998, the Treasury
} 
used multi-unit auction formats, as well as the multi-unit Vickrey (1961) auction. To explore the new effects relative to unit-demand settings, we first depart from the single-unit demands minimally by considering a flat demands environment: multi-unit demands with constant marginal utility. We allow for general distributions of bidder values, generalizing the Milgrom and Weber (1982) single-object model. We then examine additional effects introduced by decreasing marginal utility, in settings where bidders' values are symmetric and decrease linearly.

Our main findings can be summarized as follows. The uniform-price and pay-as-bid auctions, respectively, may appear to be multi-unit extensions of the second-price and first-price auctions for a single item. Nonetheless, the attractive truth-telling and efficiency attributes of the second-price auction do not carry over to the uniform-price auction; nor does the uniform-price auction, as a general theoretical matter, generate as much expected revenues as the pay-as-bid auction. ${ }^{3}$ In fact, every equilibrium of the uniform-price auction is ex post inefficient (with flat demands, this holds generically in capacities). Our Inefficiency Theorem relies on differential bid shading. This is apparent from considering the standard first-price auction: every bidder shades his bid, but with symmetric bidders and in a symmetric equilibrium, higher bids still imply higher values. In certain settings where efficiency is impossible in the uniform-price auction, full efficiency is nevertheless possible in the pay-as-bid auction. For example, with flat demands, bids for all units are shaded by identical amounts, which remains consistent with efficiency.

Considering the objectives of efficiency and revenue maximization, we find that the rank-

adopted the uniform-price design, which it still uses today, for all marketable securities. The two auction formats also have become standard designs when selling IPOs, repos, electricity, and emission permits. For instance, the European Central Bank uses auctions in refinancing (repo) operations on a weekly and monthly basis; since July 2000, these auctions have been pay-as-bid. U.K. electricity generators sell their products via daily auctions; the uniform-price format was adopted in 1990, but U.K. electricity auctions switched to the pay-as-bid price format in 2000.

${ }^{3}$ Friedman (1960) conjectured that the uniform-price auction would dominate the pay-as-bid auction in revenues. The notion that sincere bidding does not extend to the uniform-price auction where bidders desire multiple units originates in the seminal work of Vickrey (1961). Nevertheless, this analogy motivated the influential public debate between two auction formats in the context of U.S. Treasury auctions. The Joint Report on the Government Securities Market (1992, p. B-21), signed by the Treasury Department, the Securities and Exchange Commission, and the Federal Reserve Board, stated: "Moving to the uniform-price award method permits bidding at the auction to reflect the true nature of investor preferences ... In the case envisioned by Friedman, uniform-price awards would make the auction demand curve identical to the secondary market demand curve." In September 1992, the Treasury began experimenting with the uniform-price format, encouraged by Milton Friedman. Empirical evidence on the superiority of either auction format in the Treasury experiment was inconclusive (Malvey and Archibald (1998), p. 14; see also Malvey, Archibald and Flynn (1996) and Reinhart and Belzer (1996)). In switching to uniform pricing, the Treasury was apparently motivated in part by an incorrect extension to the multi-unit setting of Milgrom and Weber's (1982) result that the second-price auction generates greater revenue than the first-price auction: "One of the basic results of auction theory is that under a certain set of assumptions the revenue to the seller will be greater with uniform-price auctions than with [pay-as-bid] auctions." (Malvey and Archibald (1998), p. 3). 
ing is generally ambiguous for both criteria. ${ }^{4}$ We construct environments where the payas-bid auction dominates the uniform-price auction both in expected gains from trade and expected seller revenues, yet we construct other environments where the reverse rankings hold. We qualify this ambiguous message with two positive results. First, in auctions with symmetric bidders and flat demands, the pay-as-bid auction (as well as the Vickrey auction) dominates the uniform-price auction in both efficiency and revenues, for any fixed number of bidders. Second, in symmetric information settings with decreasing linear marginal utility, even with ex post efficient allocations, revenues can be ranked: the pay-as-bid auction dominates the Vickrey auction, which in turn dominates the uniform-price auction, for all environments where linear equilibria exist. With decreasing marginal utility, none of the multi-unit auctions considered extracts the entire surplus, even in the competitive limit (where shading is absent in the uniform-price, but not in the pay-as-bid design. Moreover, while the seller faces a trade-off between expected revenues and riskiness when selecting an auction format, this trade-off disappears in large markets. Our analysis also draws attention to the critical role of entry in the assessment of design performance.

Two modeling features of our framework are worth highlighting. First, our model allows for interdependent values. It is essential that the bidder conditions his bid on the information revealed by winning a particular quantity of the good. Extending the notions of the first-order statistic and Winner's Curse to multi-unit auction setting, we assume that winning a larger quantity of the good is worse news about the good's value, since winning more means that others do not value the good as highly as they might - an effect that we term the Generalized Winner's Curse. As a result, a rational bidder shades his bid to avoid bidding above his conditional marginal value for the good, as with the standard Winner's Curse. Henceforth, we will refer to bid shading as bidding below the bidder's conditional marginal value for the good, rather than merely as the shading that arises from Winner's Curse avoidance.

Second, obtaining a sharp ranking of multi-unit auction formats in settings with decreasing marginal utility requires strong assumptions. Indeed, our approach is motivated in part by empirical research on multi-unit auctions. As part of the challenge in this literature, obtaining predictive results in multi-unit auctions requires identifying a solution concept that addresses the multiplicity of equilibria, a problem that is understood to be endemic for uniform-price auctions. The linear equilibrium does just this for the model with diminishing linear marginal utility. ${ }^{5}$

\footnotetext{
${ }^{4}$ Important earlier work by Back and Zender (1993) in a pure common value setting demonstrated that revenues may be lower from the uniform-price auction than from a particular equilibrium of the pay-as-bid auction.

${ }^{5}$ The linear equilibrium allowed us to provide several positive results along with the general conclusions of the first part; in particular, the expected revenue ranking for all distributions that admit linear equilibria; the ex post revenue ranking for general distributions; a stochastic dominance result implying risk-revenue tradeoff for the seller; of the uniform-price and Vickrey auctions (Propositions 4, 5, 8, 9; Theorem 3). The
} 
The theorems of our paper are stated formally for static multi-unit auctions where bidders submit bid schedules, and so the theorems are most obviously applicable to sealed-bid auctions such as those for Treasury bills or electricity. However, most of our results can be adapted to any auction context where equilibria possess a uniform-price character. For example, in the simultaneous ascending auctions used for spectrum licenses, there is a strong tendency toward arbitrage of the prices for identical items. ${ }^{6}$ Similarly, consider items that are sold through a sequence of (single-item) English auctions. The declining-price anomaly notwithstanding, there is a reasonable tendency toward intertemporal arbitrage of the prices for identical items, and so a variant on our Inefficiency Theorem should typically apply.

Related literature. Wilson (1979) and subsequent authors (notably, Back and Zender (1993) and Wang and Zender (2002)) develop the continuous methodology of "share auctions" that we exploit. However, each of these papers assumes pure common values, so that allocative efficiency is never an issue - every allocation is efficient. Back and Zender (1993), as well as Wang and Zender (2002), also address the issue of revenue ranking of the uniform-price and pay-as-bid auctions for a class of functional forms. They faced the methodological limitation of comparing one equilibrium (out of a multiplicity of equilibria) of the uniform-price auction with one equilibrium of the pay-as-bid auction. By contrast, our Inefficiency Theorem is a statement about the entire set of equilibria; and our analysis of settings with diminishing linear marginal utility is based on the linear equilibrium, which is unique in both auction formats.

Noussair (1995) and Engelbrecht-Wiggans and Kahn (1998) examine uniform-price auctions where each bidder desires up to two identical, indivisible items. They find that a bidder generally has an incentive to bid sincerely on his first item but to shade his bid on the second item. Engelbrecht-Wiggans and Kahn (1998) provide a construction which is suggestive of the inefficiency and revenue results we obtain below, and offer a particularly ingenious class of examples in which bidders bid zero on the second unit with probability one. Tenorio (1997) examines a model with two bidders who each desire up to three identical items and is constrained to bid a single price for a quantity of either two or three. He finds that greater demand reduction occurs under a uniform-price auction rule than under a pay-as-bid rule.

linear equilibrium gives rise to fixed-point characterizations of price impacts. The linear equilibrium is widely used in the literature, particularly for the uniform-price auction, and has some empirical support in both formats (see Ft. 16). Our characterization of the class of distributions which admit such linear equilibrium in the discriminatory price auction is of independent interest. The assumptions on distributions (but not values) are admittedly less general, but the revenue ranking results for the linear marginal utility give a complete understanding for the class of Linear Bayesian Equilibria, which has been the focus of the theory and microstructure literature on games with demand schedules as strategies, and allow us to consistently separate the effect of decreasing marginal utility and uncertainty on bidding.

${ }^{6}$ Indeed, in the FCC's Nationwide Narrowband Auction of July 1994, similar licenses were on average priced within 0.3 percent of the mean price for that category of license, and the five most desirable licenses sold to three different bidders identically for $\$ 80$ million each. 
Bolle (1997) addresses the efficiency question which we pose here. In a framework restricted to discrete goods and to independent private values, he simultaneously and independently of our work concludes that equilibria of the uniform-price and pay-as-bid auctions are always inefficient. ${ }^{7}$

The revenue in multi-unit auctions where bidders desire multiple units of a good was studied by Engelbrecht-Wiggans (1988) and Maskin and Riley (1989), who show that the weak form of the Revenue Equivalence Theorem holds in an independent private value setting: each bidder's surplus, and hence the seller's revenue, depends only on the allocation of the goods. Auctions that result in the same allocation of goods necessarily yield the same revenue. As we indicate, however, the uniform-price, pay-as-bid, and Vickrey auctions generally assign goods differently, so the strong form of revenue equivalence fails.

Controlled field and experimental studies confirm the presence of demand reduction in the uniform-price auctions. Kagel and Levin (2001) find substantial demand reduction with uniform pricing, regardless of whether the auction was static or dynamic. Similarly, List and Lucking-Reiley (2000) find demand reduction in Internet experiments with two units and two bidders. Engelbrecht-Wiggans, List, and Lucking-Reiley (2006) conduct sportscard auctions with more than two bidders. Consistent with our results, they find that demand reduction diminishes with competition but does not vanish. Extensive literature of laboratory experiments on revenue rankings has tended to slightly favor the uniform-price auction, except when bidders' demand curves are sufficiently steep (Smith (1967, 1982)). More recently, a growing empirical literature seeks structural methods to examine bidding behavior and compare auction mechanisms (e.g., Hortaçsu and McAdams (2010), Wolak (2003, 2007), Février, Préget and Visser (2004), Armantier and Sbai (2006), Chapman, McAdams and Paarsch (2007), Hortaçsu and Puller (2008), and Kastl (2010)). The pioneering work of Hortaçsu and McAdams (2010) using Turkish data finds that the pay-as-bid auction produced more revenue, ex post, than the uniform-price auction would have, but the authors fail to reject ex ante expected revenue equality.

This paper is organized as follows. In Section 2, we develop a series of examples providing some intuition for bidding behavior and its impact on efficiency and revenues. Section 3 introduces a general model of divisible good auctions. In Section 4, we analyze the special case of constant marginal utility up to a fixed capacity. This flat demands assumption simplifies the analysis while still nesting most of the unit-demand settings that have been analyzed in the literature. We establish that, generically, all equilibria of the uniform-price auction

\footnotetext{
${ }^{7}$ Holmberg (2009) and Hasto and Holmberg (2006) study electricity markets in which the bidders can take both long (buy) and short (sell) positions in the auction, and they show that bidders prefer the uniform-price auction to the pay-as-bid auction. Other theoretical advances focused on the revenue rankings and efficiency in large, competitive markets (Swinkels (2001); Federico and Rahman (2003); Jackson and Kremer (2006)), abstracting from the strategic effects of bidders' market power (Federico and Rahman (2003) also analyze monopolistic market structures).
} 
are inefficient (Theorem 1) and that the efficiency and revenue rankings of the uniform-price and pay-as-bid auctions are ambiguous (Theorem 2). One insight from this comparison is that bidder heterogeneity matters: Equilibrium efficiency of the pay-as-bid auction relies on strong symmetry of bidder values and capacities (compare Propositions 2 and 3). Then, in Section 5, we consider bidders with diminishing linear marginal utilities in a symmetricinformation model and we examine the additional multi-unit effects that are introduced. In this setting, we establish expected revenue dominance of the pay-as-bid auction over the Vickrey and uniform-price auctions for all symmetric linear equilibria (Theorem 3) and ex post revenue dominance of the Vickrey auction over the uniform-price auction (Proposition 8). Section 6 concludes, emphasizing the practical importance of demand reduction, as seen in spectrum and electricity auctions. Appendix A contains the proofs, Appendix B offers additional examples, and Appendix $\mathrm{C}$ provides a full treatment of Example V (Section 2.2).

\section{Examples}

We illustrate the intuition of this paper with a series of simple two-bidder, two-unit examples. We first discuss how the presence of multi-unit demands alters strategic incentives in a standard asymmetric information setting. To highlight the differences relative to an auction with unit demands, we assume that each bidder has the same value for a second unit as for the first. In the second part, we discuss strategic considerations arising purely from diminishing marginal utility for the second unit.

\section{$2.1 \quad$ Flat demands}

Consider two bidders with quasilinear utilities bidding for a supply of two identical, indivisible items. Each bidder $i(i=1,2)$ has a constant marginal value $v_{i} \geq 0$ for a first and second unit (except for Bidder 1 in Example IV, who demands only a single unit). Thus, bidder $i$ paying $P_{i}$ for $q_{i}$ units receives utility $u_{i}\left(v_{i}, q_{i}, P_{i}\right)=q_{i} v_{i}-P_{i}$. The examples are:

EXAMPLE I: Each bidder $i$ has a constant marginal value, $v_{i}$, for two units, where $v_{i} \sim$ $U[0,100]$, for $i=1,2$.

EXAMPLE II: Each bidder $i$ has a constant marginal value, $v_{i}$, for two units, where $v_{1} \sim$ $U\left[0,66 \frac{2}{3}\right]$ and $v_{2} \sim U\left[0,133 \frac{1}{3}\right]$.

EXAMPLE III: Each bidder $i$ has a constant marginal value, $v_{i}$, for two units, where $v_{1} \sim$ $U[0,80]$ and $v_{2} \sim U[40,80]$.

ExAmple IV: Bidder 1 has value for only a single unit, while Bidder 2 has a constant marginal value for two units, where $v_{i} \sim U[0,100]$, for $i=1,2$.

Since the demands are flat, an auction is ex post efficient if it allocates both units to the 
bidder with the higher realization of $v_{i}$, except in Example IV, where if $v_{1}>v_{2}$, then each bidder receives one unit for efficiency.

We consider three standard multi-unit auction formats: the two formats predominantly used in practice, the uniform-price and pay-as-bid auctions; as well as the theoretical benchmark of the Vickrey auction. In each of these formats, a bidder submits a bid given by two numbers $\left(b_{i}^{1}, b_{i}^{2}\right)$, one for each item. The auctioneer ranks all four bids and awards items to the two highest bids. The formats differ in how the payment $P_{i}$ is determined.

Uniform-Price Auction: In the uniform-price auction, the monetary payment for each item is given by the highest rejected bid (i.e., the third-highest bid). We show below that the bidding strategies:

$$
b_{i}^{1}\left(v_{i}\right)=v_{i} \text { and } b_{i}^{2}\left(v_{i}\right)=0, \text { for } i=1,2,
$$

constitute a Bayesian-Nash equilibrium of the uniform-price auction in each of Examples I-IV, i.e., regardless of whether the values are drawn from symmetric or asymmetric distributions. Thus, a bidder with demand for two units behaves in this equilibrium as if he had a positive marginal value for only a single unit, and he bids his true value for that unit. ${ }^{8}$

As in the second-price auction for a single item, it is weakly dominant for both bidders to bid their true value for the first item; the first bid determines the price only when it is the third-highest bid, in which case the bidder wins zero items and the price is irrelevant to the bidder. When the first bid does not set the price, profits are maximized by making the bid compete favorably against all bids below the bidder's true value; the bidder then wins a unit only when it can profitably be won.

Now, consider bids for the second item. Given strategy (1) of bidder $j$, bidder $i$ 's bid of $b_{i}^{2}$ faces two possibilities: if $b_{i}^{2}<v_{j}$ then bidder $i$ wins one item and pays $b_{i}^{2}$; if $b_{i}^{2}>v_{j}$ then bidder $i$ wins two items and pays $v_{j}$ for each. Thus, bidder $i$ 's expected payoff from strategy $\left(v_{i}, b_{i}^{2}\right)$ is:

$$
\pi_{i}\left(v_{i}, b_{i}^{2}\right)=2 \int_{0}^{b_{i}^{2}}\left(v_{i}-p\right) d F_{j}(p)+\left(v_{i}-b_{i}^{2}\right)\left(1-F_{j}\left(b_{i}^{2}\right)\right),
$$

where $F_{j}(\cdot)$ is the distribution function of bidder $j$ 's value. Bidding more aggressively for the second item increases the probability of winning that item, while increasing the expected payment for the first item. Note that increasing the bid $b_{i}^{2}$ by a small amount $\epsilon>0$ changes the expected payoff by approximately $\left[\left(v_{i}-b_{i}^{2}\right) f_{j}\left(b_{i}^{2}\right)-\left(1-F_{j}\left(b_{i}^{2}\right)\right)\right] \epsilon$. With the uniform distribution, this effect is always negative, and so it is strictly optimal for the bidder to submit $b_{i}^{2}=0$. In other words, it is optimal for him to shade his value maximally for the

\footnotetext{
${ }^{8}$ Equilibria with this structure were discovered by Noussair (1995) and Engelbrecht-Wiggans and Kahn (1998) in closely related models.
} 
second unit, regardless of his true value. ${ }^{9}$

The described Bayesian-Nash equilibrium is ex post inefficient. Even though one of the bidders has a strictly higher value for both items with probability one in Examples I-III and in half of all realizations of value in Example IV, the two items are always allocated to different bidders. Moreover, the uniform-price auction performs very poorly in relation to revenues: for each realization of values, the third-highest bid is zero and, hence, the revenues are zero as well.

In addition to this "zero-revenue" equilibrium, there is also an efficient equilibrium of the uniform-price auction for Examples I-III. It is obtained by embedding the truthtelling equilibrium of the second-price auction for a single item into the multi-unit game. If a bidder's opponents bid $b_{i}^{1}\left(v_{i}\right)=v_{i}=b_{i}^{2}\left(v_{i}\right)$, then it is a best response for the bidder to do the same. However, as we show in Section 4, the existence of this second equilibrium crucially requires that the agents' capacities to absorb units (in the Examples I-III, equal to 2) must be identical and supply must be an integer multiple of the common capacity. If this is not the case, Theorem 1 in Section 4 establishes that an efficient equilibrium does not exist.

Thus, Examples I-III also illustrate the pervasive multiplicity of equilibria of the uniformprice auction, first observed by Wilson (1979). Example IV provides not only an instance where inefficiency is mandatory, but is in an unusual class of settings where equilibrium of the uniform-price auction is unique - see Example B1 of Appendix B.

Pay-as-Bid Auction: In the pay-as-bid auction, the monetary payment for each item coincides with the corresponding (winning) bid for that item. In particular, with flat bids, the bid for one item does not affect the payment for the other item, and submitting the same value for both items (i.e., flat bids) is an equilibrium. To construct such a flat-bid equilibrium, denote the common bid of bidder $j$ by $b_{j}\left(v_{j}\right)=b_{j}^{1}\left(v_{j}\right)=b_{j}^{2}\left(v_{j}\right)$. If bidder $j$ follows the flat-bid strategy while bidder $i$ bids $\left(b_{i}^{1}, b_{i}^{2}\right)$, then $i$ 's utility is:

$$
\pi_{i}\left(b_{i}^{1}, b_{i}^{2}\right)=\sum_{k=1,2}\left(v_{i}-b_{i}^{k}\right)\left(F_{j}\left(b_{j}^{-1}\left(b_{i}^{k}\right)\right)\right) .
$$

This utility can be maximized pointwise and is symmetric with respect to both items. ${ }^{10}$ In

\footnotetext{
${ }^{9}$ In the uniform-price auction, the bid on the pivotal unit determines the price on all other units won. This creates an incentive to bid less than the true value on later units to reduce the price on the earlier units. With discrete goods, this intuition suggests that the bidder will bid his true value on his first unit demanded, but strictly less than his true value on all subsequent units. With divisible goods, it suggests further that a bidder's submitted demand curve will take on the qualitative features of a monopolist's marginal-revenue curve: at zero quantity, the demand curve and the bid curve (marginal revenue curve) intersect, but at all positive quantities, the bid curve (marginal revenue curve) lies strictly below the true demand curve. We establish these features of bid shading for discrete values and divisible decreasing marginal utilities.

${ }^{10}$ The separability of expected profits from the two items implies that the Bayesian-Nash equilibrium of the first-price auction for a single item can be embedded into the the pay-as-bid auction with symmetric bidders. The strategies and per-item revenues are then the same as in the first-price auction.
} 
the symmetric case, the distributions of values are identical and the first-order condition $F_{j}\left(b_{i}^{-1}\left(b_{i}^{k}\right)\right)=\left(v_{i}-b_{i}^{k}\right) f_{j}\left(b_{i}^{-1}\left(b_{j}^{k}\right)\right), k=1,2$, has a symmetric solution $b_{i}(\cdot)=b_{j}(\cdot)$. The auction then efficiently allocates both items. In the asymmetric case, the equilibrium strategies are asymmetric and the outcome is inefficient.

Vickrey Auction: In the benchmark multi-unit Vickrey auction, the payment for bidder $i$ 's first and second items (if won) is the sum of the first- and the second-highest rejected bids reported by bidders $j \neq i$. A bidder has no impact on his own payments and bidding $b_{i}^{1}\left(v_{i}\right)=b_{i}^{2}\left(v_{i}\right)=v_{i}$ for $i=1,2$ is weakly dominant for each bidder and constitutes a Bayesian-Nash equilibrium, which is ex post efficient.

Combining the above observations with further analysis in Section 4 and numerical calculations reported in Tables 1 and 2, we can reach the following conclusions:

- In symmetric Example I, each of the three auction formats has an efficient equilibrium, for which revenues and surplus are the same;

- In asymmetric Example II, which generalizes a single-unit example of Maskin and Riley (2000), the uniform-price and Vickrey auctions have efficient equilibria; the pay-as-bid auction's equilibrium, while inefficient, raises higher revenues;

- In asymmetric Example III, revenue maximization coincides with efficiency - see the first paragraph of the proof of Theorem 2-and, consequently, the efficient equilibrium of the uniform-price and Vickrey auctions dominate the inefficient equilibrium of the pay-as-bid auction in both revenues and surplus;

- In Examples I-III, the uniform-price auction also displays an equally plausible second equilibrium, which generates zero revenues and markedly reduced surplus; and

- In Example IV, the zero-revenue equilibrium is the unique Bayesian-Nash equilibrium of the uniform-price auction, and the pay-as-bid auction's equilibrium dominates it in both revenues and surplus.

Table 1 summarizes numerical calculations of expected revenues in the constructed BayesianNash equilibria of the three standard auction formats:

Table 1. Expected Revenues in Two-Item Auctions

\begin{tabular}{r|r||cccc}
\hline \multicolumn{1}{c||}{ Revenues } & & Example I & Example II & Example III & Example IV \\
\hline \hline PAY-AS-BID & & 66.67 & 61.19 & 61.99 & 30.04 \\
\hline UNIFORM-PRICE & Zero-Revenue Equilibrium & 0 & 0 & 0 & 0 \\
\hline UNIFORM-PRICE & Efficient Equilibrium & 66.67 & 55.56 & 73.33 & - \\
\hline VICKREY & & 66.67 & 55.56 & 73.33 & 33.33 \\
\hline
\end{tabular}


Table 2 summarizes numerical calculations of expected surplus in the constructed BayesianNash equilibria of the three standard auction formats:

Table 2. Expected Surplus in Two-Item Auctions

\begin{tabular}{r|r||cccc}
\hline Surplus & & Example I & Example II & Example III & Example IV \\
\hline \hline PAY-AS-BID & & 133.33 & 141.68 & 95.22 & 115.03 \\
\hline UNIFORM-PRICE & Zero-Revenue Equilibrium & 100 & 100 & 100 & 100 \\
\hline UNIFORM-PRICE & Efficient Equilibrium & 133.33 & 144.44 & 126.67 & - \\
\hline VICKREY & & 133.33 & 144.44 & 126.67 & 116.67 \\
\hline
\end{tabular}

\subsection{Diminishing marginal utility ${ }^{11}$}

In our next example, we argue that allowing for diminishing marginal utility qualitatively changes bidding incentives in multi-unit auctions. To this end, we modify Example I as follows.

EXAMPLE V: Each bidder $i$ has decreasing marginal utility, with $v_{i}^{1}=v_{i}$ for the first unit and $v_{i}^{2}=\rho v_{i}$ for the second unit, where $v_{i} \sim U[0,100]$, for $i=1,2$ and $\rho \in(0,1)$ capturing the "diminution" of marginal utility.

Example $\mathrm{V}$ directly nests Example I as its limit when $\rho \rightarrow 1$. In both examples, bidders are symmetric. Similarly to the original example, we restrict attention to symmetric and monotone equilibria in which each buyer submits two bid functions $\left(b^{1}(\cdot), b^{2}(\cdot)\right)$ that are weakly increasing in $v_{i}$, once continuously differentiable, and satisfy $b^{1}(\cdot) \geq b^{2}(\cdot)$. The detailed derivation of equilibria, and arguments supporting existence and uniqueness for the three auction formats are given in Appendix C.

Uniform-Price Auction: In the uniform-price auction, a lower $\rho$ reduces incentives to bid for the second unit relative to flat demands, and bids given by (1) constitute a Bayesian Nash equilibrium also when $\rho<1$. The equilibrium is ex post inefficient and yields zero revenue. Contrary to the flat demand settings, bidding one's true values $\left(b_{i}^{1}, b_{i}^{2}\right)=\left(v_{i}, \rho v_{i}\right)$, $i=1,2$, is not an equilibrium. With truth telling, for realizations of values of opponent $j$, $v_{j} \in\left(\rho v_{i}, v_{i}\right)$, the uniform-price auction assigns to bidder $i$ one unit for which he pays $b_{i}^{2}$. For other realizations of $v_{j}$, bidder $i$ either does not win any unit or does not determine the price. It follows that bidder $i$ has a strict incentive to reduce the second bid $b_{i}^{2}$ below his true value $\rho v_{i}$. In Appendix $\mathrm{C}$, we demonstrate that the zero-revenue equilibrium (1) is unique within the class of symmetric monotone equilibria.

Pay-as-Bid Auction: By standard arguments (as in the first-price auction), in a symmetric

\footnotetext{
${ }^{11}$ The results of Section 2.2 (and Appendix C) were originally developed in Weretka (2014).
} 
monotone equilibrium, bid functions $b^{1}(\cdot), b^{2}(\cdot)$ are strictly increasing. Bidder $i$ observes $v_{i}$ and submits $\left(b_{i}^{1}, b_{i}^{2}\right)$ satisfying $b_{i}^{1} \geq b_{i}^{2}$. With no benefit from overbidding for the first unit at $v_{i}=100, b^{1}(100)=b^{2}(100)=\bar{b}$, and both bids are from some interval $b \in[0, \bar{b}]$. Let $\phi^{1}(\cdot), \phi^{2}(\cdot)$ denote corresponding inverses of $b^{1}(\cdot), b^{2}(\cdot)$, satisfying $\phi^{2}(\cdot) \geq \phi^{1}(\cdot)$. The marginal bid distribution of buyer $j$ for units $k=1,2$ is $\operatorname{Pr}\left[b^{k}\left(v_{j}\right) \leq b\right]=F\left[\phi^{k}(b)\right]=\frac{\phi^{k}(b)}{100}$. Bidder $i$ wins two units if the second bid exceeds $j^{\prime} s$ bid for the first unit, $b_{i}^{2}>b^{1}\left(v_{j}\right)$. The probability of this event is $F\left[\phi^{1}\left(b_{i}^{2}\right)\right]$, and he wins one unit if $b_{i}^{1}>b^{2}\left(v_{j}\right)$ and $b_{i}^{2}<b^{1}\left(v_{j}\right)$, which happens with probability $F\left[\phi^{2}\left(b_{i}^{1}\right)\right]-F\left[\phi^{1}\left(b_{i}^{2}\right)\right]$. Thus, $i^{\prime} s$ net expected utility is given by:

$$
\begin{aligned}
\pi_{i}\left(b_{i}^{1}, b_{i}^{2}\right) & =F\left[\phi^{1}\left(b_{i}^{2}\right)\right]\left(v_{i}+\rho v_{i}-b_{i}^{1}-b_{i}^{2}\right)+\left(F\left[\phi^{2}\left(b_{i}^{1}\right)\right]-F\left[\phi^{1}\left(b_{i}^{2}\right)\right]\right)\left(v_{i}-b_{i}^{1}\right)= \\
& =F\left[\phi^{1}\left(b_{i}^{2}\right)\right]\left(\rho v_{i}-b_{i}^{2}\right)+F\left[\phi^{2}\left(b_{i}^{1}\right)\right]\left(v_{i}-b_{i}^{1}\right) .
\end{aligned}
$$

The net utility functions consist of two separate components, each depending on the bid for one of the two units. The first order conditions and the uniform distribution jointly imply for any $b \in[0, \bar{b}]$ :

$$
\begin{aligned}
{\left[\phi^{1}(b)\right]^{\prime}\left(\rho v_{i}-b\right) } & =\phi^{1}(b), \\
{\left[\phi^{2}(b)\right]^{\prime}\left(v_{i}-b\right) } & =\phi^{2}(b) .
\end{aligned}
$$

By equilibrium symmetry, the optimal bids satisfy $b_{i}^{1}=b^{1}\left(v_{i}\right)$ and $b_{i}^{2}=b^{2}\left(v_{i}\right)$, and hence

$$
\left[\phi^{1}(b)\right]^{\prime}=\frac{\phi^{1}(b)}{\rho \phi^{2}(b)-b},\left[\phi^{2}(b)\right]^{\prime}=\frac{\phi^{2}(b)}{\phi^{1}(b)-b} .
$$

Following the steps analogous to the derivation of equilibrium in the first-price auction with asymmetric bidders, one can solve the system of differential equations and obtain equilibrium bids by inverting $\phi^{k}(\cdot)$. In particular, bidding $\left(b^{1}(\cdot), b^{2}(\cdot)\right)$ by each agent, where

$$
\begin{aligned}
& b^{1}\left(v_{i}\right)==\frac{100^{2} \rho^{2}}{\rho^{2}-1} \frac{1}{v_{i}}\left(1-\sqrt{1+\frac{1-\rho^{2}}{100^{2} \rho^{2}}\left(v_{i}\right)^{2}}\right), \\
& b^{2}\left(v_{i}\right)=\frac{100^{2} \rho}{1-\rho^{2}} \frac{1}{v_{i}}\left(1-\sqrt{1-\frac{1-\rho^{2}}{100^{2}}\left(v_{i}\right)^{2}}\right),
\end{aligned}
$$

constitutes a symmetric Bayesian-Nash equilibrium. Bid functions for various values of $\rho$ are depicted in Figure 1. In Appendix $\mathrm{C}$, we demonstrate that equilibrium is unique in the symmetric monotone class.

In contrast to flat demands, decreasing marginal utility introduces asymmetry across units, and bidders shade their values differently for both units. As a result, for some re- 

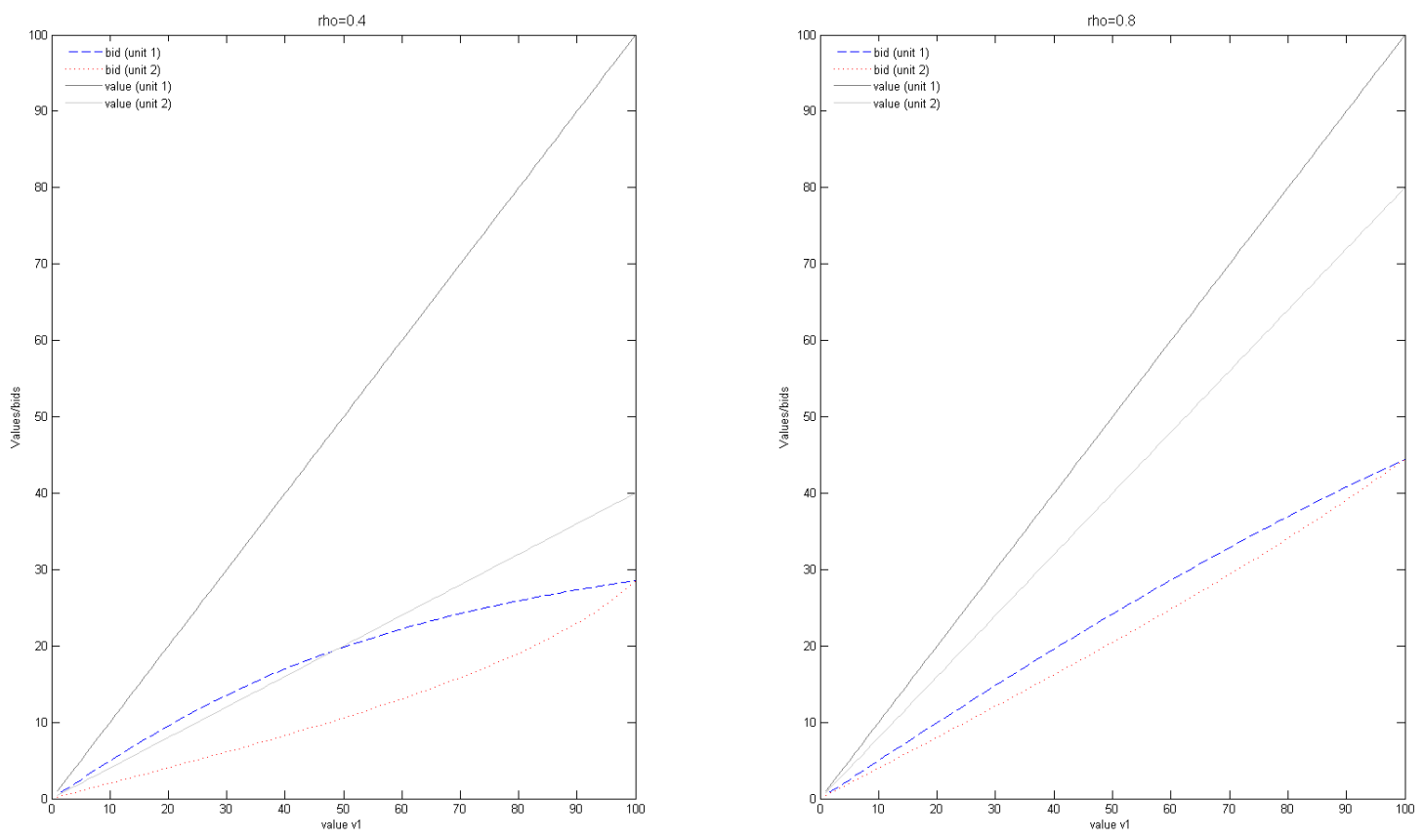

Figure 1: BIDS IN THE PAY-AS-BID AUCTION

alizations of values, units are not assigned to agents with the highest values. The shading behavior, however, differs qualitatively from what we have seen before. In the uniform-price auction, the equilibrium bid function is steeper than the true demand curve. By contrast, in the pay-as-bid auction, the equilibrium bid function is flatter than the true demand curve: agents bid below their true values for both units, with bid shading larger for the first unit. Expected revenue is strictly positive for all $\rho \in(0,1)$. The limit of bids (3) as $\rho \rightarrow 1$ converges to the equilibrium strategies in Example I; at the opposite extreme, as $\rho \rightarrow 0$, there is no competition for the second unit and revenues (from both units) converge to zero. Example $\mathrm{V}$ continuously extends the predictions of the flat-demands model to settings with diminishing marginal utility.

Vickrey Auction: In the multi-unit Vickrey auction with diminishing marginal utility, all agents bidding their true values continues to be an equilibrium. As a result, the expected surplus is maximized; it is monotonic in $\rho$, increasing from 100 to 133.33 as $\rho$ goes from 0 to 1. Expected revenues are also monotonic in $\rho$, increasing from 0 to 66.67 as $\rho$ goes from 0 to 1 ; they are below the revenues of the pay-as-bid auction for $\rho \in(0,1)$, and they converge to the same revenues as the pay-as-bid auction at the end points.

Numerical calculations for different values of $\rho$ are depicted in Figure 2. Panel (a) of Figure 2 compares the expected surplus in the unique symmetric monotone Bayesian-Nash equilibria of each auction format, while panel (b) compares the expected revenues. This suggests the 

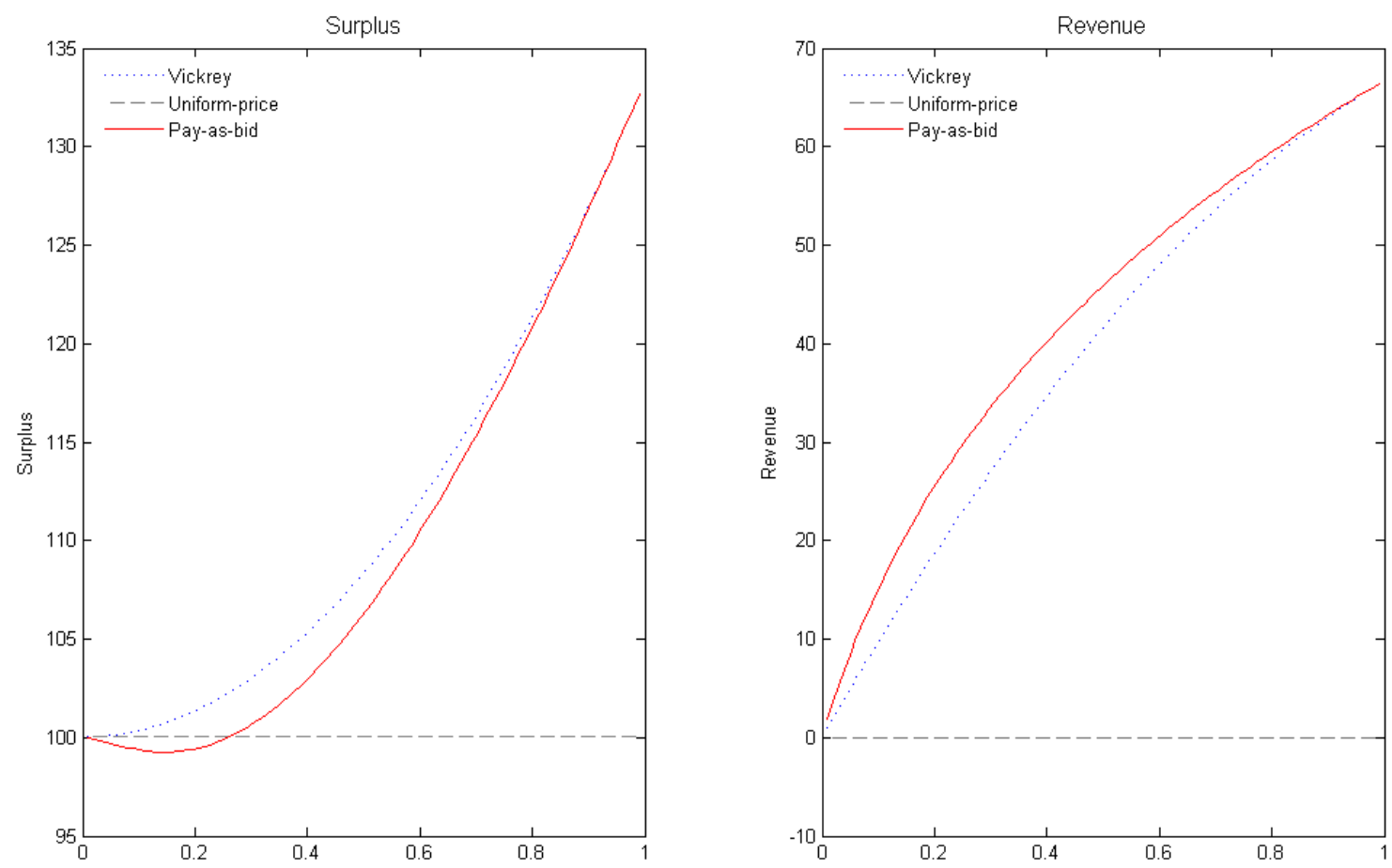

Figure 2: Surplus And Revenue in the three AuCtion formats

following conclusions when there is diminishing marginal utility (Example V), as compared to flat demands (Example I):

- The isolated instances in which truth telling is an equilibrium of the uniform-price auction are eliminated;

- Symmetric monotone equilibria are unique in the three auction formats;

- Equilibrium bid shading is differential in the uniform-price and pay-as-bid auctions;

- Bid shading is increasing in the uniform-price auction and decreasing in the pay-as-bid auction, in the number of units won;

- The pay-as-bid and uniform-price auctions are inefficient;

- Efficiency rankings are ambiguous: the pay-as-bid auction dominates (is dominated by) the uniform-price auction for large (small) values of $\rho$;

- The pay-as-bid auction gives higher expected revenues than the Vickrey auction, which in turn dominates the uniform-price auction, for all values of $\rho \in(0,1)$; 
- For $\rho \rightarrow 0$, all auctions give the maximal surplus of 100 and zero revenue, while for $\rho \rightarrow 1$, the surplus and revenue converge to those in Example I (and, specifically for the uniform-price auction, the zero-revenue equilibrium).

While the examples of this Section have been special, they are representative of more general results that will follow. Auctions in settings with flat demands are studied more generally in Section 4, and auctions in settings with perfectly divisible goods and linearly decreasing marginal utility are studied in Section 5. Multiplicity of equilibrium is endemic to multi-unit auctions, making efficiency and revenue comparisons of auction formats potentially problematic. Diminishing marginal utility introduces further strategic aspects. A recurring theme of our paper is to find compelling ways to make comparisons possible.

\section{Model}

We make the following general assumptions. Quantity $Q$ of a perfectly-divisible good is sold to $I$ bidders. Each bidder receives a private signal $s_{i} \in[0,1]$ of his value $v_{i}$ before bids are submitted; this signal will be referred to as the bidder's type. Let $s \equiv\left\{s_{i}\right\}_{i=1}^{I}$ and let $s_{-i} \equiv\left\{s_{j}\right\}_{j \neq i}$. Types are drawn from the joint distribution $F$ with support $[0,1]^{I}$ and finite density $f$ that is strictly positive on $(0,1)^{I}$. While distribution function $F$ is commonly known to bidders, the realization $s_{i}$ is known only to bidder $i$. A bidder $i$ with value $v_{i}$ consuming $q_{i}$ and paying $P_{i}$ has a payoff $u_{i}\left(v_{i}, q_{i}, P_{i}\right)$, where $v_{i}=v_{i}(s)$. The seller's valuation for the good is 0 .

An assignment of the good auctioned among bidders $Q^{*}(s) \equiv\left(Q_{1}^{*}(s), \ldots, Q_{I}^{*}(s)\right)$ is said to be ex post efficient if each unit goes to the bidder who values it the most:

$$
Q^{*}(s) \equiv \arg \max _{Q_{1}(s), \ldots, Q_{I}(s)}\left\{\sum_{i=1}^{I} u_{i}\left(v_{i}(s), Q_{i}(s), 0\right) \mid \sum_{i=1}^{I} Q_{i}(s) \leq Q\right\} .
$$

The seller uses a conventional auction to allocate the good. In a conventional auction, bidders simultaneously and independently submit bids and the items are awarded to the highest bidders. In the formal analysis we assume that, having observed his signal $s_{i}$, each bidder $i$ submits a bid function $b_{i}\left(\cdot, s_{i}\right):[0, Q] \rightarrow[0, \infty)$ expressing the value $b_{i}$ bid for any quantity $q$. We require the function $b_{i}$ to be right-continuous at $q=0$, left-continuous at all $q \in(0, Q]$ and weakly decreasing. The market-clearing price $\bar{p}$ is then set at the highest rejected bid,

$$
\bar{p}=\min \left\{p \mid \sum_{i=1}^{I} b_{i}^{-1}(p) \leq Q\right\}
$$


where $b_{i}^{-1}$ is bidder $i$ 's demand function constructed by inverting his bid function. ${ }^{12}$ If $\sum_{i} b_{i}^{-1}\left(\bar{p}, s_{i}\right)=Q$, then each bidder $i$ is assigned a quantity of $Q_{i} \equiv b_{i}^{-1}\left(\bar{p}, s_{i}\right)$. If $\sum_{i} b_{i}^{-1}\left(\bar{p}, s_{i}\right)>$ $Q$, then the aggregate demand curve is flat at $\bar{p}$, and some bidders' demands at $\bar{p}$ will need to be rationed. ${ }^{13}$ The pricing rule $P_{i}$ depends on the auction:

Uniform-Price Auction: Each bidder $i$ assigned $Q_{i}$ pays the market clearing price $\bar{p}$ for each of the $Q_{i}$ units obtained; $i$ 's total payment is $P_{i}=Q_{i} \bar{p}$.

Pay-as-Bid Auction: Each bidder $i$ assigned $Q_{i}$ pays his winning bids; $P_{i}=\int_{0}^{Q_{i}} b_{i}\left(y, s_{i}\right) d y$.

Note that most other sealed-bid auction formats in the literature (most conspicuously, the multi-unit Vickrey auction) also satisfy the definition of a conventional auction.

Finally, the equilibrium concept used in this paper is the usual Bayesian-Nash Equilibrium, which comprises a profile of bid functions, $b_{i}\left(\cdot, s_{i}\right)$, for every type of every bidder which are mutual best responses.

\section{Constant Marginal Values}

In this section, we study bidders with constant marginal values for the good, up to fixed capacities, i.e. "flat demands".

\subsection{Assumptions}

In the analysis of the flat demands model, we normalize $Q=1$ without loss of generality. Each bidder $i$ can consume any quantity $q_{i} \in\left[0, \lambda_{i}\right]$, where $\lambda_{i} \in(0,1)$ is a capacity; formally, we assume that utility from consuming quantities $q_{i}>\lambda_{i}$ is negative. To make the problem nontrivial, we require that there be competition for each quantity of the good: for each $i, \sum_{j \neq i} \lambda_{j} \geq 1$. One can interpret $q_{i}$ as bidder $i$ 's share of the total quantity and $\lambda_{i}$ as a

\footnotetext{
${ }^{12}$ In section with flat demands, an inverse bid $b_{i}^{-1}\left(\cdot, s_{i}\right)$ is constructed from the bid function as follows. Given fixed $s_{i}$, let $\Gamma=\left\{\left(q, b_{i}\left(q, s_{i}\right)\right) \mid q \in\left[0, \lambda_{i}\right]\right\} \cup\left\{(0, \bar{P}),\left(\lambda_{i}, 0\right)\right\}$, where $\lambda_{i}$ is the capacity of bidder $i$ (defined in Section 4.1), capacities denote the graph of $b_{i}(q)$ and the two additional points which say that, at a sufficiently high price $\bar{P}$, the bidder demands nothing, and at a price of zero, the bidder demands his optimal quantity (denoted $\lambda_{i}$ ). Take the closure of $\Gamma$, and then fill vertically all the discontinuities of the demand curve, and call the result $\Gamma^{\prime}$. Define a weakly-decreasing correspondence $\gamma_{i}(p)=\left\{q \mid(q, p) \in \Gamma^{\prime}\right\}$, and define function $b_{i}^{-1}\left(p, s_{i}\right)$ to be the selection from $\gamma_{i}(p)$ which is left-continuous at $p=\bar{P}$ and right-continuous at all $p \in[0, \bar{P})$. Since each $b_{i}^{-1}\left(\cdot, s_{i}\right)$ is weakly decreasing, and since the construction for inverting bid functions imposes that $b_{i}^{-1}(0)=\lambda_{i}$ and $b_{i}^{-1}(\bar{P})=0$, observe that the market-clearing price $\bar{p}$ exists and is unique, and $\bar{p} \in(0, \bar{P})$.

${ }^{13}$ If there is just a single bidder whose demand curve is flat at $\bar{p}$, then this bidder's quantity is reduced by $\sum_{i} b_{i}^{-1}\left(\bar{p}, s_{i}\right)-Q$. If there are multiple bidders with demand curves flat at $\bar{p}$, then quantity is allocated by proportionate rationing. For our purposes, the specific tie-breaking rule will not matter, since with probability one, there is at most a single bidder with flat demand at $\bar{p}$. Define bidder $i$ 's incremental demand at $\bar{p}$ as $\Delta_{i}(\bar{p}) \equiv b_{i}^{-1}\left(\bar{p}, s_{i}\right)-\lim _{p \downarrow \bar{p}} b_{i}^{-1}\left(p, s_{i}\right)$. Then, bidder $i$ is awarded an amount $b_{i}^{-1}\left(\bar{p}, s_{i}\right)-$ $\left.\left(\sum_{i} b_{i}^{-1}\left(\bar{p}, s_{i}\right)\right)-Q\right) \Delta_{i}(\bar{p}) / \sum_{i} \Delta_{i}(\bar{p})$.
} 
quantity restriction. For example, in the U.S. Treasury auctions, a bidder's net long position, including both pre-auction trading and the auction award, cannot exceed $35 \%$. The FCC spectrum auctions have had similar quantity restrictions.

Bidder $i$ has a constant marginal value $v_{i} \in[0,1]$ for the good up to the capacity $\lambda_{i}$, and the bidder's utility is $u_{i}\left(v_{i}, q_{i}, P_{i}\right)=q_{i} v_{i}-P_{i}$, for $q_{i} \in\left[0, \lambda_{i}\right]$. The relationship between types $s_{i} \in[0,1]$ and values $v_{i}(\cdot, \cdot)$ is common knowledge among bidders, and is assumed to satisfy the following:

Assumption 1 (Value Monotonicity) Function $v_{i}\left(s_{i}, s_{-i}\right)$ is strictly increasing in $s_{i}$, weakly increasing in each component of $s_{-i}$, and continuous in all its arguments.

Assumption 2 (Types Rank VAlues) $s_{i}>s_{j} \Rightarrow v_{i}(s)>v_{j}(s)$.

The model generalizes that of independent private values in two ways: values may depend on the private information of others, and a bidder's private information need not be independent of the private information of others. The types rank values assumption deliberately excludes a pure common value model, since in that case any assignment respecting the capacities $\lambda_{i}$-and hence any auction that does not have a reserve price and that does not force bidders to buy more than they want - is efficient. ${ }^{14}$ Note that the above assumptions imply that any two ex-post efficient assignments are equal with probability one.

A critical element in the analysis of auctions for a single good is the first-order statistic. If $Y_{i}=\max \left\{s_{j} \mid j \neq i\right\}$ is the highest signal of bidders other than $i$, then bidder $i$ receives the good in the efficient assignment only if $s_{i} \geq Y_{i}$. In $m$-unit auctions where each bidder can win at most one unit, the $m^{\text {th }}$-order statistic serves the analogous role. However, when analyzing general multi-unit auctions the order statistics by themselves are inadequate: the quantity won by a bidder confers additional information. We thus appropriately generalize the first-order statistic notions to a multi-unit auction.

Definition. Fix an efficient assignment $Q^{*}$. For any $s_{-i} \in[0,1]^{I-1}$ and $q \in\left(0, \lambda_{i}\right]$, define $\tau_{i}^{q}\left(s_{-i}\right) \equiv \inf \left\{s_{i} \in[0,1]: Q_{i}^{*}\left(s_{i}, s_{-i}\right) \geq q\right\}$, the minimal signal of bidder $i$ such that this bidder is assigned at least $q$ items in the efficient assignment $Q^{*}$. Let $F_{i}^{q}(y \mid x)=\operatorname{Pr}\left\{\tau_{i}^{q}\left(s_{-i}\right) \leq\right.$ $\left.y \mid s_{i}=x\right\}$ be the c.d.f. of statistic $\tau_{i}^{q}\left(s_{-i}\right)$ conditional on $i$ 's own signal, and let $f_{i}^{q}(y \mid x)$ denote the associated density function. Let $w_{i}^{q}(x, y)=\mathbb{E}\left[v_{i}\left(s_{i}, s_{-i}\right) \mid s_{i}=x, \tau_{i}^{q}\left(s_{-i}\right)=y\right]$ be an expected value conditional on own signal and statistic $\tau_{i}^{q}\left(s_{-i}\right)$, and (if defined) let $w_{i}^{+}(x, x) \equiv \lim _{q \downarrow 0} w_{i}^{q}(x, x)$.

Note that, with probability one, $Q^{*}(s)$ is defined uniquely by equation (4). Furthermore, $\tau_{i}^{q}\left(s_{-i}\right)$ is defined uniquely for every $s_{-i} \in(0,1)^{I-1}$ and $q \in\left(0, \lambda_{i}\right]$. We will henceforth assume that the primitives of the model have been specified such that $F_{i}^{q}(y \mid x), f_{i}^{q}(y \mid x)$, and $w_{i}^{q}(x, y)$, when needed, are mathematically well-defined functions, and such that $w_{i}^{q}(x, y)$ is continuous in $(x, y)$.

\footnotetext{
${ }^{14}$ Section 5 studies a special case of the pure common value model.
} 
Essentially all of the previous auction literature has made assumptions that imply the presence of the Winner's Curse, the notion that winning is "bad news": a bidder's expected value conditional on winning is less than or equal to his unconditional expected value. In the single-good case, the standard assumptions postulate that each bidder's expected value from the good $\tilde{v}_{i}(x, y) \equiv E\left[v_{i} \mid s_{i}=x, Y_{i}=y\right]$ is strictly increasing in $x$ and weakly increasing in $y$ (Milgrom and Weber, 1982, p. 1100). Our value monotonicity Assumption 1 implies that $w_{i}^{q}(x, y)$ is strictly increasing in $x$ and weakly increasing in $y$ for all bidders $i$ and quantity levels $q$. To extend the Winner's Curse concept to the multi-unit auction setting, we also need to capture the idea that winning a larger quantity is "worse news" than winning a smaller quantity. We thus assume the following.

Assumption 3 (Generalized Winner's Curse) A multi-unit auction environment exhibits the Generalized Winner's Curse if, for all bidders $i, w_{i}^{q}(x, x)$ is weakly decreasing in $q$. Note that this assumption implies that $w_{i}^{+}$is well-defined for all bidders $i$.

\section{$4.2 \quad$ Efficiency}

We begin our analysis by noting that, in any of the considered auction formats, an equilibrium can be efficient only if the bids are flat.

Proposition 1 (EFfICIENT BIDs) If a Bayesian-Nash equilibrium of a conventional auction attains ex post efficiency then all bidders use symmetric, monotonic, flat bid functions: there exists a strictly increasing function $\phi:[0,1] \rightarrow[0,1]$ such that $b_{i}\left(q, s_{i}\right)=\phi\left(s_{i}\right)$ for all bidders $i=1, \ldots, I$, for all quantities $q \in\left[0, \lambda_{i}\right]$, and for almost every type $s_{i} \in[0,1]$. Moreover, in the uniform-price auction, every bidder $i$ uses the symmetric, flat bid function $b_{i}\left(q, s_{i}\right)=\phi\left(s_{i}\right)=w^{+}\left(s_{i}, s_{i}\right)$, for every type $s_{i} \in[0,1]$ and every quantity $q \in\left[0, \lambda_{i}\right]$.

To see heuristically why efficiency requires flat bidding, consider a symmetric equilibrium where $b_{i}=b_{j}$ for all bidders $i, j$. Efficiency requires that the bidder with the highest value, say bidder $i$, receives quantity $\lambda_{i}$. Thus $b_{i}\left(q, s_{i}\right) \geq b_{j}(0, y)=b_{i}(0, y)$ for all bidders $j \neq i$ and signals $y<s_{i}$. The monotonicity and left-continuity of $b_{i}$ allow us to conclude that $b_{i}$ is flat. We provide a complete proof in the Appendix.

Uniform-Price Auction: Next, we develop the main insight of Section 4: all equilibria of the uniform-price auction are inefficient. We then finish the equilibrium analysis by looking at the efficiency of pay-as-bid auctions.

Theorem 1 (Generic Inefficiency of Uniform-Price Auction): Consider a flatdemand setting that exhibits the Generalized Winner's Curse. There exists an ex post efficient equilibrium of the uniform-price auction if, and only if, $\lambda_{i}=\lambda$ for all $i, 1 / \lambda$ is an integer, and $w_{i}^{+}(x, x)=w_{j}^{+}(x, x)$ for all $i, j$ and $x$. 
The intuition behind Theorem 1 is that bidders have market power in the uniform-price auction. If a bidder has a positive probability of influencing price in a situation where the bidder wins a positive quantity, then the bidder has incentives to shade his bid. In particular, if a bidder cannot be pivotal for small quantities then he bids his expected values for them. If the same bidder is pivotal with positive probability for large quantities then he shades his bid for such quantities. Consequently, his bid cannot be flat, and by the preceding proposition, the equilibrium is not efficient. We show that such a bidder exists, unless $\lambda_{i}=\lambda$ for all $i$ and $1 / \lambda$ is an integer.

The logic is as follows. By Proposition 1, in an efficient equilibrium each bidder $i$ expects other bidders $j \neq i$ to submit flat bids. Thus, bidder $i$ 's bids for sufficiently small quantities are never pivotal: for any subset of other bidders $I^{\prime} \subset\{1, \ldots, I\}-\{i\}$ whose combined capacity satisfies $\sum_{j \in I^{\prime}} \lambda_{j}<1$, adding a sufficiently small quantity $q_{i}$ to the combined capacity of bidders in $I^{\prime}$ does not reverse the strict inequality, $\sum_{j \in I^{\prime}} \lambda_{j}+q_{i}<1$. Thus, bids $b_{i}\left(q, s_{i}\right)$ for small quantities $q$ never determine the market-clearing price. Analogous to the reasoning for the second-price auction of a single item, it will then be optimal for bidder $i$ to maximize the probability of winning in all events in which the expected value, conditional on winning, exceeds the payment. Hence bidder $i$ bids $b_{i}\left(q, s_{i}\right)=w_{i}^{q}\left(s_{i}, s_{i}\right)$ for all small $q$. This part of the argument relies on the assumption of a Generalized Winner's Curse. ${ }^{15}$

Furthermore, by Proposition 1, in an efficient equilibrium the bid function is constant for all quantities up to capacity, and hence the necessary condition for efficiency is

$$
b_{i}\left(q, s_{i}\right)=w_{i}^{+}\left(s_{i}, s_{i}\right) \text { for } q \in\left[0, \lambda_{i}\right] .
$$

This condition is generically violated in a Bayesian-Nash Equilibrium. In the Appendix, we demonstrate that flat bid $b_{i}\left(q, s_{i}\right)=w_{i}^{+}\left(s_{i}, s_{i}\right)$ is not a best response for the bidder with the greatest capacity (say, bidder 1 ), unless $\lambda_{i}=\lambda$ for all $i=1, \ldots, I$ and $1 / \lambda$ is an integer. Specifically, there exists a subset of bidders other than $i=1, J \subset\{2, \ldots, I\}$, for which $\sum_{j \in J} \lambda_{j}<1$ and $\sum_{j \in J} \lambda_{j}+\lambda_{1}>1$. Then, for a quantity threshold $\bar{L}_{1} \equiv 1-\sum_{j \in J} \lambda_{j}$, bidding strategy

$$
\hat{b}_{1}\left(q, s_{1}\right)=\left\{\begin{array}{l}
w_{1}^{+}\left(s_{1}, s_{1}\right) \text { for } q \in\left[0, \bar{L}_{1}\right] \\
\beta \quad \text { for } q \in\left(\bar{L}_{1}, \lambda_{1}\right]
\end{array}\right\},
$$

for $\beta$ less than but sufficiently close to $w_{1}^{+}\left(s_{1}, s_{1}\right)$, yields a strictly higher payoff than strategy (5). This is so because with positive probability the signals of all bidders from set $J$ are higher than $s_{1}$ while the signals of the remaining bidders are lower than $s_{1}$, and bidder 1 wins $\bar{L}_{1}$ units at price $\beta$. Such an event gives bidder $i=1$ an incentive to shade his bid for

\footnotetext{
${ }^{15}$ In the absence of the Generalized Winner's Curse, $w_{i}^{q}\left(s_{i}, s_{i}\right)>w_{i}^{+}\left(s_{i}, s_{i}\right)$ for some $q \in\left(0, \lambda_{i}\right]$. Because bids are constrained to be weakly decreasing in quantity, this violation of the Generalized Winner's Curse would imply that bidder $i$ might want to bid more than $w_{i}^{+}\left(s_{i}, s_{i}\right)$ at some small $q \in\left(0, \lambda_{i}\right]$ in order to be able to bid higher than $w_{i}^{+}\left(s_{i}, s_{i}\right)$ at some large $q \in\left(0, \lambda_{i}\right]$.
} 
sufficiently large quantities, $q \in\left(\bar{L}_{1}, \lambda_{1}\right]$.

For an integer $1 / \lambda$ with $\lambda_{i}=\lambda$, the proof of inefficiency does not go through. In this special case, a bidder affects price only when he wins nothing, and bidding expected value conditional on winning $w_{i}^{q}\left(s_{i}, s_{i}\right)=w_{i}^{+}\left(s_{i}, s_{i}\right)$ for all $q_{i}$ is a best response. Hence, bids (5) for all $i$ constitute an equilibrium. Moreover, if $w_{i}^{+}\left(s_{i}, s_{i}\right)$ is identical for all bidders, efficiency is achieved.

We see that efficiency of the uniform-price auction requires a substantial amount of symmetry in the model. In environments with interdependent values, the condition that $w_{i}^{+}(x, x)=w_{j}^{+}(x, x)$ for any $i, j$ is unlikely to be satisfied without symmetry of value functions, capacities, and distribution of types. By imposing several symmetry assumptions, we obtain an environment that satisfies the Generalized Winner's Curse, and we can apply Theorem 1 to determine when there exists an efficient equilibrium.

Corollary 1 (Symmetric Interdependent Values Model) Consider a flat demands setting that additionally satisfies:

(i) $v_{\pi_{1}}\left(s_{\pi_{1}}, \ldots, s_{\pi_{I}}\right)=v_{1}\left(s_{1}, \ldots, s_{I}\right)$ for any permutation $\pi_{1}, \ldots, \pi_{I}$ of $1, \ldots, I$;

(ii) $F\left(s_{\pi_{1}}, \ldots, s_{\pi_{I}}\right)=F\left(s_{1}, \ldots, s_{I}\right)$ for any permutation $\pi_{1}, \ldots, \pi_{I}$ of $1, \ldots, I$;

(iii) $\left(s_{1}, \ldots, s_{I}\right)$ are affiliated random variables; and

(iv) $\lambda_{i}=\lambda$, for all $i(i=1, \ldots, I)$.

Then, there exists an ex post efficient equilibrium of the uniform-price auction if, and only if, $1 / \lambda$ is an integer.

The above corollary includes, as a special case, the independent private values model in which individual values (or, equivalently, individual signals) are drawn from the same distribution. The independent private values environment satisfies the Generalized Winner's Curse even if the agents' values are drawn from different distributions, and thus the following further corollary obtains.

Corollary 2 (Independent Private Values Model) Consider a flat demands model, with $v_{i}\left(s_{i}, s_{-i}\right)=s_{i}$ and $\lambda_{i} \equiv \lambda$ for each $i=1, \ldots, I$, and with independent but not necessarily identically distributed $F_{i}(\cdot)$. There exists an ex post efficient equilibrium of the uniform-price auction if, and only if, $1 / \lambda$ is an integer.

Pay-as-Bid Auction: We now establish that in some situations in which efficiency is impossible in the uniform-price auction, full efficiency is nevertheless possible in the pay-as-bid auction. The intuition is straightforward: the inefficiency result in the uniform-price auction is driven by the incentive for demand reduction due to price impact, in that a bidder who shades his bids on subsequent units saves money on the purchase of earlier units. By contrast, this incentive does not exist in the pay-as-bid auction with flat demands; a bidder 
who reduces his bid for subsequent units (but holds his bids constant on earlier units) does not realize any savings on his purchase of earlier units.

This is analogous to the situation of a monopolist deciding how much to produce. Recall that the uniform-price auction is often referred to as a "nondiscriminatory auction" while the pay-as-bid auction is referred to as a "discriminatory auction." Just as monopoly without price discrimination leads to social inefficiency while a monopolist with perfect price discrimination may realize all gains from trade, a nondiscriminatory auction will lead to inefficiency but a discriminatory auction has the possibility of efficiency. The nondiscriminating monopolist's marginal revenue curve lies strictly below his demand curve, except at zero quantity; the perfectly discriminating monopolist's marginal revenue curve may actually coincide with his demand curve. We therefore obtain supply reduction in the former but not necessarily in the latter situation.

To construct an efficient Bayesian-Nash equilibrium of the pay-as-bid auction, consider bidders that have independent private values $v_{i}(s)=s_{i}$ and are ex ante symmetric: their signals $s_{i}$ are i.i.d., and their capacities $\lambda_{i}=\lambda$ are equal for all $i$. Let $U_{i}\left(v_{i}\right)$ denote the interim expected utility of bidder $i$, and let $Q_{i}\left(v_{i}\right)$ denote the interim expected quantity received by bidder $i$ in an efficient direct mechanism. Let $m$ be the greatest integer less than $1 / \lambda$, let $v_{(m)}^{-i}$ denote the $m^{t h}$ order statistic of signals of all bidders except $i$, and let $F_{(m)}^{-i}(\cdot)$ denote its distribution function. Observe that efficiency requires that bidder $i$ must obtain $\lambda$ units of the good if $v_{i}>v_{(m)}^{-i}, 1-m \lambda$ units of the good if $v_{(m+1)}^{-i}<v_{i}<v_{(m)}^{-i}$, and 0 units of the good if $v_{i}<v_{(m+1)}^{-i}$. Thus,

$$
Q_{i}\left(v_{i}\right)=\lambda F_{(m)}^{-i}\left(v_{i}\right)+(1-m \lambda)\left[F_{(m+1)}^{-i}\left(v_{i}\right)-F_{(m)}^{-i}\left(v_{i}\right)\right]
$$

Since the interim expected utility of the zero type must equal zero, the usual incentivecompatibility argument implies that $U_{i}\left(v_{i}\right)=\int_{0}^{v_{i}} Q_{i}(x) d x$. Now suppose that an efficient equilibrium of the pay-as-bid auction exists. By Proposition 1, each bidder must use a flatbid function almost everywhere: $b_{i}\left(q, v_{i}\right)=\phi_{i}\left(v_{i}\right)$. Using this bid function, an alternative way to calculate the interim expected utility of bidder $i$ is $U_{i}\left(v_{i}\right)=Q_{i}\left(v_{i}\right)\left[v_{i}-\phi_{i}\left(v_{i}\right)\right]$. Combining the two expressions for utility gives the equilibrium bid. In the appendix, we build on this argument to prove the following:

Proposition 2 (EFficient PAY-AS-BID AUCtion) If bidders have independent private values $v_{i}(s)=s_{i}$ and are ex ante symmetric, i.e., if their signals $s_{i}$ are i.i.d., and their capacities $\lambda_{i}=\lambda$ are equal, then

$$
b_{i}\left(q, v_{i}\right)=\phi_{i}\left(v_{i}\right)=v_{i}-\frac{\int_{0}^{v_{i}} Q_{i}(x) d x}{Q_{i}\left(v_{i}\right)}
$$

constitutes an ex-post efficient equilibrium of the pay-as-bid auction. 
This positive result does not mean that the pay-as-bid auction should be preferred to uniform pricing. It is well known that a first-price auction for a single indivisible item does not admit an efficient equilibrium except in special settings. If bidders' values are random variables that are not identically distributed, then any equilibrium of the first-price auction will typically be inefficient. These considerations from the first-price auction carry over to the current context; the assumption in Proposition 2 that each bidder's marginal value, $v_{i}$, is drawn from the same distribution should be viewed as essential. Proposition 3 treats the case of asymmetric bidders and easily obtains a negative result.

Proposition 3 (Inefficient PAy-AS-BID Auction) If bidders'values are independent but not identically distributed or if their capacities are unequal, then generically there does not exist an ex post efficient equilibrium of the pay-as-bid auction.

\subsection{Ambiguous Rankings of Conventional Auctions}

Early discussions of U.S. Treasury auctions conjectured that the uniform-price auction is superior to the pay-as-bid auction when selling multiple units in terms of both revenue and efficiency. We have shown above that this conjecture, which derives largely from the analysis of auctions in which bidders have tastes for only a single unit, is flawed. In uniform-price auctions, rational bidders strategically submit lower unit prices for larger quantities than for smaller quantities, even when demands are flat, adversely affecting allocative efficiency. By contrast, the pay-as-bid auction need not suffer from demand reduction, enabling it to yield full efficiency in some situations where the uniform-price auction cannot.

We shall now show that in some circumstances the pay-as-bid auction also raises more revenue than the uniform-price auction. Theorem 2 demonstrates that the efficiency and revenue rankings of the two auction formats are both ambiguous. We establish this theorem via two positive results which identify two environments where revenue maximization coincides with full efficiency. Our construction is based on the principle that in any flat-demands environment, revenues are maximized (subject to no reserve price) by allocating items to the bidders in descending order of their "marginal revenues," $M R_{i}\left(v_{i}\right)=v_{i}-\frac{1-F_{i}\left(v_{i}\right)}{f_{i}\left(v_{i}\right)}$, up to their capacities $\lambda_{i}$ (see Ausubel and Cramton (1999)). We say that the marginal revenues are monotonic in values if $M R\left(v_{i}\right)>M R\left(v_{j}\right) \Longleftrightarrow v_{i}>v_{j}$.

In the first environment, the pay-as-bid auction attains efficiency while the uniform-price auction cannot. Consequently, it has the feature that the pay-as-bid auction dominates with respect to both revenues and efficiency.

Proposition 4 (Dominance OF PAY-AS-BID AUCtion) Consider any symmetric flatdemands environment in which the marginal revenues are monotonic in values and the bidders' capacities satisfy $\lambda_{i}=\lambda$, for all $\mathrm{i}=1, \ldots$, I. Then, pay-as-bid auction dominates 
uniform-price in terms of both revenue and efficiency. Furthermore, the dominance is strict if $1 / \lambda$ is not an integer.

In the second environment, the uniform-price auction attains efficiency while the payas-bid auction cannot. ${ }^{16}$ Here, the uniform-price auction dominates with respect to both revenues and efficiency.

Proposition 5 (Dominance of Uniform-Price Auction) Consider a flat-demand model in which the bidders' capacities satisfy $\lambda_{i}=1$ for all $i=1, \ldots, I$. Let $F$ be a cdf with support $[\underline{v}, \bar{v}]$, let $f$ be its density, and let its marginal revenue be monotonic in values. Suppose that $\underline{v}_{i} \in[\underline{v}, \bar{v})$ and each bidder $i=1, \ldots, I$ has his value drawn independently from distribution $F_{i}=\frac{F(v)-F\left(\underline{v}_{i}\right)}{1-F\left(\underline{v}_{i}\right)}$ on $\left[\underline{v}_{i}, \bar{v}\right]$. Then, uniform-price auction dominates pay-as-bid in terms of both revenue and efficiency. Furthermore, the dominance is strict if there are some $i, j$ such that $F_{i} \neq F_{j}$.

We prove this proposition in Appendix A. Let us illustrate the forces behind the dominance of the uniform-price auction in the following example. Consider the asymmetric single-item ${ }^{17}$ auction environment in which $v_{1} \sim U\left[\eta_{1}, 1\right], v_{2} \sim U\left[\eta_{2}, 1\right], \ldots, v_{I} \sim U\left[\eta_{I}, 1\right]$, where $0 \leq \eta_{1}<\eta_{2}<\ldots<\eta_{I}<1$. Observe by an easy calculation that marginal revenues are monotonic in values, and so revenues are maximized by allocating the item to the bidder with the highest value. The uniform-price auction now collapses to the second-price auction; bidding one's true value, which is the unique equilibrium in undominated strategies, attains full efficiency and consequently maximizes revenues. We will have established that the equilibrium of the uniform-price auction dominates the equilibrium of the pay-as-bid auction, with respect to both efficiency and revenues, provided we can show that the equilibrium of the first-price auction with these distributions is inefficient. This is demonstrated as follows. For $I=2$ bidders, suppose that the first-price auction has an efficient equilibrium in undominated strategies. For efficiency, bidder 2 must use a monotonic bidding strategy, and all types $v_{1}<\eta_{2}$ of bidder 1 must win the auction with zero probability. It follows that, for any $\epsilon>0$, type $\eta_{2}+\epsilon$ of bidder 2 must bid at least $\eta_{2}-\epsilon$. Otherwise, types $v_{1} \in\left(\eta_{2}-\frac{\epsilon}{2}, \eta_{2}\right)$ of bidder 1 could profitably deviate by bidding $\eta_{2}-\epsilon$. Define $p=\frac{\frac{1}{2}\left(\eta_{1}+\eta_{2}\right)-\eta_{1}}{1-\eta_{1}}$, the probability

\footnotetext{
${ }^{16}$ For simplicity of exposition, we state this and the next proposition assuming no type of bidder $i$ has values between 0 and $\underline{v}_{i}$. This violates our modeling assumptions that bidders draw signals from $[0,1]^{I}$ and the mapping from signals to values is continuous. One can adapt the propositions to our modeling assumptions by shifting a small mass $\epsilon$ of each bidder's types to have values in $\left[0, \underline{v}_{i}\right)$. The desired revenue ranking will still go through provided that the mass $\epsilon$ is sufficiently small.

${ }^{17}$ The essential aspects of this counterexample and the preceding proposition do not require a single-unitdemand environment. Alternatively, we could assume that the bidders' capacities satisfy $\lambda_{i}=\lambda$, for all $i=$ $1, \ldots, I$, and $1 / \lambda=M$, any integer. However, the efficient equilibrium of the uniform-price auction would generally no longer be unique; see, for example, the discussion in Section 2.
} 
that bidder 1's type is less than $\frac{1}{2}\left(\eta_{1}+\eta_{2}\right)$. By bidding $\frac{1}{2}\left(\eta_{1}+\eta_{2}\right)$, every type of bidder 2 can assure himself a payoff of at least $\frac{1}{2}\left(\eta_{2}-\eta_{1}\right) p>0$. Consequently, for $\epsilon$ sufficiently small, type $\eta_{2}+\epsilon$ of bidder 2 does not optimally bid at least $\eta_{2}-\epsilon$, a contradiction. We conclude that there is no efficient equilibrium of the first-price auction. A similar argument can be made with more than two bidders.

The above two propositions imply our second major result.

Theorem 2 (Ambiguous RANkings) The efficiency and revenue rankings of the uniformprice and pay-as-bid auctions are inherently ambiguous.

To summarize, in general, the revenue and efficiency rankings of the two commonly-used auction formats are ambiguous. However, in all settings with symmetric buyers that we study, the pay-as-bid auction dominates the uniform-price auction.

\section{Diminishing Marginal Values}

Apart from affecting bidder's incentives in the presence of asymmetric information, as analyzed so far, another aspect of multi-unit demands not present in auctions of unit demands is the possibility of decreasing marginal utility, which itself introduces new effects, even in settings where agents' valuation functions are identical. To study such effects we now allow bidders to have marginal values that are decreasing in the quantity received. We assume that the bidders have linear marginal utilities with the same slope and value for the good $\left(s_{i}=v\right.$ for all $\left.i\right)$. We examine linear equilibria, in which bids $b_{i}(\cdot, v): \mathbb{R} \rightarrow \mathbb{R}_{+}$are linear in quantity and value. ${ }^{18}$ The uniqueness of the linear equilibrium allows us to compare the auction formats in a consistent way. ${ }^{19}$

\footnotetext{
${ }^{18}$ The strategy space is not restricted to the class of linear bids; rather, in a linear equilibrium, it is optimal for a bidder to submit a linear bid, given that the other bidders play linear strategies. For the uniform-price mechanism, the linear (Bayesian) Nash equilibrium has been widely used in modeling financial, electricity, and other oligopolistic markets. Hortaçsu's (2002) study of the Turkish Treasury auction and Hortaçsu and Puller's (2008) study of spot market for electricity in Texas find that linear equilibrium provides a good description of the data. Analysis based on non-linear equilibria is developed in Glebkin and Rostek (2014).

${ }^{19}$ With unbounded support of supply, the linear equilibrium of the uniform-price auction is unique within a large class of equilibria studied by Klemperer and Meyer (1989). They study a model of a procurement auction with an exogenous downward-sloping demand, and show that when utilities are quadratic and uncertainty has unbounded support, Nash equilibrium in the uniform-price auction is unique in the set of strategies that are piecewise differentiable functions. Their result applies directly to our uniform-price model (with a vertical supply); thus, in our analysis for Generalized Pareto distributions with $\xi>0$, the uniform-price equilibrium is unique within a large class. For distributions with $\xi<0$, the set of Nash equilibria in the uniform-price auction is not determinate (but the linear equilibrium is unique). When the utilities are not quadratic, apart from the result by Klemperer and Meyer (1989) that the set of equilibria is connected, no results are available in the literature about the determinacy of equilibrium in the uniform-price auction. For the pay-as-bid auction, Back and Zender (1993), Wang and Zender (2002), and Pycia and Woodward (2014) prove the uniqueness of equilibrium.
} 
We identify the class of distributions that admits a linear equilibrium and provide revenue rankings for all distributions in this class. Decreasing marginal utility changes bidding incentives and equilibrium properties: Bidders shade their bids even if they have no private information; in particular, a traditional Bertrand-style argument does not apply and no auction format allows the seller to extract full surplus, even in the limit as the number of bidders grows to infinity. Moreover, even though equilibria are ex post efficient (i.e., $Q(s)=Q^{*}(s)$ ), seller revenue varies across auction formats. In particular, with any finite number of bidders, the pay-as-bid auction brings strictly higher expected revenue than the uniform-price auction.

\subsection{Assumptions}

Each bidder $i$ 's marginal utility is affine with common slope $\rho>0$ and intercept $v \in \mathbb{R}$, that is $\partial u\left(q_{i}\right) / \partial q_{i}=v-\rho q_{i}$ where $u\left(q_{i}\right)$ is the quadratic utility of bidder $i$. The value $v$ is random and commonly known to all bidders (i.e., $s_{i}=v$ for all $i$ ), but not the seller. Bidders are uncertain about the supply $Q$ being auctioned. The joint c.d.f., $F(v, Q)$ of the value $v$ and supply $Q$ - which can be correlated - is common knowledge and has non-degenerate support $F(\cdot \mid v)$. We make the usual assumption for the quadratic model that for all values of $v$ and $Q$ in the support of $F$, the bidders prefer more of the good rather than less; that is, bidders are not satiated. This last assumption implies that the support of $Q$ is compact for any $v .^{20}$

\subsection{Linear Equilibrium}

If the marginal utility was constant, bidding $b_{i}\left(q_{i}, v\right)=v$ would be a linear Nash equilibrium in both uniform-price and pay-as-bid auctions. In such an equilibrium, bidders would not shade their bids, and both auctions would be revenue maximizing; hence, they would also be revenue equivalent. None of these predictions obtains when marginal utility is decreasing, $\rho>0$.

Our derivation exploits the following feature of linear equilibrium with downward-sloping demands: Given a profile of bid functions $b_{j}(\cdot, v), j \neq i$, bidder $i$ trades against an upwardsloping, linear (residual) supply $p=x+\mu_{i} q_{i}$ where the intercept $x=x(Q)$ is a deterministic function of value $v$ and quantity $Q$. Market clearing implies that $q_{i}+\sum_{j \neq i} b_{j}^{-1}(p, v)=$ $Q$, and with symmetric bids $b_{j}=b_{j^{\prime}}$ the slope of the residual supply is given by $\mu_{i}=$ - $(1 /(I-1)) \partial b_{j}(\cdot, v) / \partial q_{j}$ (Propositions 6 and 7 below establish that the linear equilibrium is symmetric in both the uniform-price and pay-as-bid auctions). For bidder $i$, the distribution of the intercept $x$ derived from $F(\cdot \mid v)$ and $\mu_{i}$ contains all of the payoff-relevant information about the strategies of other bidders.

\footnotetext{
${ }^{20}$ The non-satiation assumption is not needed for any of the equilibrium characterization in Section 5.2. We use it only in Proposition 6 in Section 5.3.
} 
Uniform-Price Auction: The first-order condition equates marginal utility with marginal payment at each realization of supply; that is, order shading is

$$
v-\rho q_{i}-b_{i}=\mu_{i} q_{i}
$$

Aggregating the bids of bidders other than $i$ gives $i$ 's residual supply, the slope of which-i's price impact - can be characterized as

$$
\mu_{i}=\left(\sum_{j \neq i}\left(\mu_{j}+\rho\right)^{-1}\right)^{-1}
$$

The unique, symmetric solution to $I$ equations (9) gives the equilibrium price impact of bidder $i$ equal to $\mu_{i}=\rho /(I-2)$, for $I>2 .{ }^{21}$ Proposition 6 characterizes equilibrium bids.

Proposition 6 (Equilibrium in Uniform-Price Auction) Suppose $I>2$. In the unique linear equilibrium, the strategy of each bidder is

$$
b_{i}\left(q_{i}, v\right)=v-\left(\frac{I-1}{I-2}\right) \rho q_{i}
$$

Note that, with decreasing marginal utility, the equilibrium bids remain optimal ex post, when supply is known. ${ }^{22}$ By best-responding with a downward-sloping bid, a bidder effectively conditions on the realization of supply, $Q$, thereby hedging away supply uncertainty. Furthermore, for any $q_{i}$ the bid $b_{i}\left(q_{i}, v\right)$ affects a bidder's payoff only for the realization of $Q=I q_{i}$.

In contrast to the flat demands model - in which the winning bids do not affect the equilibrium price unless capacities are heterogeneous, or $1 / \lambda$ is not an integer, or supply is sufficiently large - with decreasing marginal utility, the winning bids affect the revenue irrespective of the level of supply. In linear equilibrium, bid shading $v-\rho q_{i}-b_{i}=q_{i} \mu_{i}$ is increasing in quantity, and the corresponding bid function (10) is steeper than marginal

\footnotetext{
${ }^{21}$ Wilson's (1979) characterization of equilibrium in the uniform-price auction includes the case of $I=2$ while assuming constant marginal utility. Non-existence of equilibrium with two bidders for decreasing marginal utility is standard (e.g., Kyle (1989)).

${ }^{22}$ Proposition 6 and its argument extend to a more general model with private information and interdependent values, a setting we analyzed in 2007 draft of the second of the papers subsumed by the present merged work. For independent private values, no restrictions on (nondegenerate) distributions of values are required. For interdependent values, Rostek and Weretka (2012) characterize the necessary and sufficient condition for the ex post property of equilibrium (see also Vives (2011). With private information, the equilibrium assignment is generically inefficient due to the increasing-in-quantity bid shading: a high-value bidder is shading more than a low-value bidder at the market-clearing price. Since quantity is assigned based on the bids, the high-value bidder wins too little and the low-value bidder wins too much, relative to the efficient allocation. That is, uniform pricing gives large bidders incentives to make room for smaller bidders. In multi-unit auctions - with flat or decreasing marginal utility - it's not shading per se but differential bid shading that gives rise to inefficiency.
} 
utility. Note also that the payment structure of the uniform-price mechanism together with the decreasing marginal utility imply that even if the bidders were not to shade their bids, they would retain a strictly positive surplus.

Pay-as-Bid Auction: Unlike the uniform-price auction (and the Vickrey auction), while a downward-slopping bid function allows conditioning on the realization of $Q$ in the pay-as-bid auction, linear equilibrium in the pay-as-bid auction is not an ex-post equilibrium. The value of bid $b_{i}\left(q_{i}, v\right)$ affects the payment for all realizations of supply larger than $Q$. Thus, the distribution of supply matters for trade-offs and hence for optimal bids.

Let $G(\cdot)$ denote the c.d.f. and $g(\cdot)$ the density function of the distribution of per capita supply $Q / I$, derived from the supply distribution $F(\cdot \mid v)$ (in the notation we suppress the dependence of $G$ and $g$ on $v$ ). The inverse hazard rate of the equilibrium quantity is defined as $h(\cdot) \equiv(1-G(\cdot)) / g(\cdot)$. When written to reveal the structure of bid shading and the role of market power, the first-order condition is

$$
v-\rho q_{i}-b_{i}=h\left(q_{i}\right)\left[\mu_{i}-\partial b_{i}(\cdot) / \partial q_{i}\right]
$$

For any $q_{i}$, the marginal benefit - measured as the marginal utility net of the bid obtained in state $Q=q_{i} I$-is equal to the cost of increased payments in all states with higher realization of supply. In the Euler equation (11), the cost is thus weighted by the inverse hazard rate $h(\cdot)$, which captures probabilistic importance of higher realizations of supply.

The dependence of the optimal bid on the distribution of equilibrium quantity raises the question of which distributions $F(\cdot \mid v)$ admit linear best responses. Since in a linear equilibrium $\mu_{i}$ and $\partial b_{i}(\cdot) / \partial q$ are constants, the Euler equation (11) defines a linear schedule only if $h(\cdot)$ is linear. Lemma 1 characterizes the class of all distributions $F(\cdot \mid v)$ that exhibit a linear inverse hazard rate $h(\cdot)$.

Lemma 1 (LINEARITY OF $h(\cdot)$ ) The inverse hazard rate $h(\cdot)$ of per capita supply $Q / I$ is linear if, and only if, $F(\cdot \mid v)$ belongs to the class of Generalized Pareto distributions.

The c.d.f. of a Generalized Pareto distribution is given by $F(Q \mid v)=1-\left(1+\xi \frac{Q-\alpha}{\sigma I}\right)^{-\frac{1}{\xi}}$, where $\xi \in \mathbb{R}$ is the shape parameter, $\alpha \in \mathbb{R}$ is the location parameter, and $\sigma I>0$ is the scale parameter. The per-capita supply $Q / I$ is then distributed according to Generalized Pareto with the inverse hazard rate $h(q)=\sigma-\xi \alpha+\xi \frac{Q}{I}$. The Generalized Pareto class encompasses distributions with decreasing $(\xi<0)$, constant $(\xi=0)$, and increasing $(\xi>0)$ inverse hazard ratios; and has a lower bound of support given by $\alpha .^{23}$

\footnotetext{
${ }^{23}$ Whenever $\xi<0$, the support also has an upper bound, equal to $-(I-\xi) / \xi$. Among distributions with compact support, $\xi=-1$ corresponds to a uniform distribution; for $\xi=0$ (exponential distribution) and $\xi>0$ (the class of Pareto distributions), the support is unbounded. Conveniently, the linearity of an inverse hazard ratio is preserved under additive (i.e., changing the location) or positive multiplicative (i.e., changing the scale) transformations of a random variable.
} 
Order shading in the pay-as-bid auction can now be understood through the properties of the supply distributions. Namely, order shading inherits monotonicity in quantity through the inverse hazard ratio and is decreasing, constant, and increasing in quantity for $\xi<0$, $\xi=0$, and $\xi>0$, respectively. By contrast to the uniform-price auction, the bid in the pay-as-bid auction is shaded at zero quantity, and for all distributions with compact support $(\xi<0)$, the bid coincides with the marginal utility at the upper end of the support, where the externality on the payments in higher states vanishes. In the uniform-price auction, there is no shading at zero quantity, and bids are strictly below the marginal utility at the upper-end quantity. Moreover, order shading in the pay-as-bid auction need not be increasing in quantity. Except when $\xi=0$ (exponential distribution), the Generalized Pareto class induces bids that can be flatter $(\xi<0)$ or steeper $(\xi>0)$ than the marginal utility, which reflects the decreasing and increasing with quantity, respectively, relative importance of higher states.

Observe that when the support of the equilibrium quantity is bounded away from 0 , the optimal bid is flat for small quantities and decreasing for large quantities, which is inconsistent with linear bidding. To rule out flat-bid parts, we set the lower bound of the support of $Q$ to zero.

Assumption 4 (Generalized Pareto Distribution: Location Parameter) For any $v, F(\cdot \mid v)$ is a Generalized Pareto distribution with location parameter $\alpha=0$.

Without imposing bid symmetry, price impact in the pay-as-bid auction is

$$
\mu_{i}=\left(\sum_{j \neq i}\left(\frac{\xi \mu_{j}}{1-\xi}+\frac{\rho}{1-\xi}\right)^{-1}\right)^{-1} .
$$

Unlike in the uniform-price auction (Equation (9)), the price impact of bidder $i$ can depend negatively on the price impact of other bidders; this occurs when $\xi<0$. With higher market power, other bidders are induced to shade more, which makes their inverse bids more elastic, thereby reducing the price impact of bidder $i$. To close the model, we determine equilibrium $\mu_{i}$ from the first-order condition and bid symmetry, $\mu_{i}=\rho /(I(1-\xi)-1)$. Proposition 7 characterizes equilibrium bids. ${ }^{24}$

Proposition 7 (Equilibrium in Pay-As-Bid Auction) Suppose $\xi<(I-1) / I$. In the

\footnotetext{
${ }^{24}$ This strategic interdependence explains why, in the pay-as-bid auction, a linear equilibrium exists even with two bidders. However, when $\xi$ exceeds $(I-1) / I$, linear equilibrium fails to exist because of the amplification of price impacts, similar to their amplification in the uniform-price auction. The non-satiation assumption implies that $\xi<0$, but our equilibrium characterization remains valid for nonnegative $\xi$. The characterization of the set of (possibly non-linear) Nash equilibria by Wang and Zender (2002) is provided for a class of supply distributions that is a strict subset of ours. We focus on linear equilibria and Proposition 7 characterizes equilibrium bids in all environments that admit linear equilibria.
} 
unique linear equilibrium, the strategy of bidder $i$ is

$$
b_{i}\left(q_{i}, v\right)=v-\frac{(I-1) \rho}{I(1-\xi)-1}\left(q_{i}+\frac{I}{I-1} \sigma\right) .
$$

By Proposition 7, bid shading in the pay-as-bid auction depends on the distribution of supply. Bid shading is proportional to the inverse hazard rate, and unlike in the case of flat demands (Proposition 2) shading can decrease or increase in quantity, as determined by the sign of parameter $\xi$. With compact support, $\xi<0$ and the bidding involves no shading at the upper end of the support of the per-capita supply. At this quantity, there is no negative effect on the payments for higher realizations of supply, and the bid coincides with the marginal value.

With decreasing marginal utility, bidders shade their value regardless of auction size, including in the limit as $I \rightarrow \infty$; this contrasts with the uniform-price auction. Although in all auction formats considered bidders have no impact on equilibrium price in the competitive auction limit (Equations (9) and (12)), uncertainty still affects order shading in the pay-as-bid auction (Equation (11)), as the bids still determine the payments for the items won. Observe further that, in markets with $\xi<0$, equilibrium converges more slowly to the price-taking limit in the pay-as-bid than in the uniform-price auction. The reduction of price impact via aggregation brought about by additional participants in the pay-as-bid auction is partially offset by the steepening of individual bids.

\subsection{Revenue Rankings}

This section presents comparative analysis of revenue in the uniform-price, pay-as-bid, and the Vickrey auctions against the benchmark of total social surplus. ${ }^{25}$ In the Vickrey auction, for the $q^{t h}$ unit, bidder $i$ is charged the reported marginal value of the item by other bidders if $q$ units are reallocated efficiently to other bidders. Thus, the total Vickrey payment of bidder $i$ is given by the opportunity cost to others and corresponds to the area below his residual supply. We examine strategic and competitive auctions, and consider risk-averse as well as risk-neutral preferences of the bidders and of the seller. Assumption 4 is maintained.

Theorem 3 establishes expected revenue rankings: Even though the optimal bidding in the pay-as-bid auction depends on the supply distribution, the comparison of the mechanisms does not. Let $R^{U}, R^{D}$, and $R^{V}$ denote the equilibrium revenues in the uniform-price,

\footnotetext{
${ }^{25}$ In our setting, the (sealed-bid) Vickrey auction is equivalent to the ascending-bid clinching auction proposed by Ausubel (2004). Note also that the total surplus can be fully extracted. Recall that $s_{i}=v$ for all $i$, and consider the following mechanism. Bidders report types $\bar{v}_{1}, \bar{v}_{2}, \ldots, \bar{v}_{I}$. Bidders with the highest reports, $\bar{v}_{i}=\max \left(\bar{v}_{1}, \bar{v}_{2}, \ldots, \bar{v}_{I}\right)$, receive $Q / \bar{I}$ units, where $\bar{I} \geq 1$ is the number of the bidders with the maximal bid, and they pay their reported surplus $\bar{v}_{i} Q \bar{I}-\frac{1}{2} \rho(Q / \bar{I})^{2}$. Bidders with lower reports receive and pay zero. By a Bertrand-type argument, the bidders bid truthfully in the unique Nash equilibrium, and the seller extracts the entire surplus.
} 
pay-as-bid (discriminatory), and Vickrey auction, respectively, and let TS be the total social surplus, all in per-capita terms.

Theorem 3 (Expected Revenue Ranking) In the unique linear equilibrium, for any $v$,

$$
\mathbb{E}(T S \mid v)>\mathbb{E}\left(R^{D} \mid v\right)>\mathbb{E}\left(R^{V} \mid v\right)>\mathbb{E}\left(R^{U} \mid v\right)
$$

whenever equilibria exist.

Consistent with a conjecture often invoked in the literature, the uniform-price design fosters more aggressive bidding in all the environments considered (see the proof of the theorem). Nevertheless, the benefit from smaller expected bid shading is not sufficient for the uniform-price to outperform the pay-as-bid auction in terms of seller's revenue. Dominance of the pay-as-bid auction with symmetric bidders is consistent with Proposition 4 and Example $\mathrm{V}{ }^{26}$

The revenue dominance of the Vickrey auction over the uniform-price auction can be strengthened: the Vickrey auction is preferred by the seller regardless of the realization of $v$ and $Q$, and, hence, regardless of the seller's risk attitude.

Proposition 8 (Ex Post Revenue Ranking) For any realization of $v$ and $Q$, the seller's revenue satisfies $T S \geq R^{V} \geq R^{U}$, and both inequalities are strict whenever $Q>0$. In particular, for any strictly increasing utility function of the seller $\bar{u}(\cdot)$, in the unique linear equilibrium,

$$
\mathbb{E}(\bar{u}(T S) \mid v)>\mathbb{E}\left(\bar{u}\left(R^{V}\right) \mid v\right)>\mathbb{E}\left(\bar{u}\left(R^{U}\right) \mid v\right)
$$

Since the equilibria of the Vickrey auction and the uniform-price auction are both ex post equilibria, the ex post dominance of the Vickrey auction over the uniform-price auction does not depend on the distributional assumptions. Furthermore, the dominance is consistent with Example V. In contrast to the Vickrey and uniform-price formats, the seller's preference for the pay-as-bid does depend on his risk attitude as we show in Proposition 9 below.

As the number of bidders increases, the expected revenue increases both absolutely and as as a fraction of the expected total surplus $E(T S \mid v)$. The ranking from Theorem 3 must hold at least weakly in the limit as $I \rightarrow \infty$ (a competitive limit). In fact, if we increase the number of bidders while keeping the distribution of per capita supply fixed, then the three auctions yield the same expected revenue in the competitive limit. Nevertheless, the common limit

\footnotetext{
${ }^{26}$ In a study of Turkish Treasury auctions, Hortaçsu and McAdams (2010) found that the pay-as-bid auction leads to higher revenues than the revenue obtainable from the Vickrey auction, which the authors attributed to the allocational inefficiency in a pay-as-bid auction with heterogenous bidders. Our result shows that the pay-as-bid auction brings higher revenue than the Vickrey auction even when the allocation is efficient in both.
} 
revenue does not extract the total surplus of $E(T S \mid v)$; fraction $\sigma \rho /(v(1-2 \xi)-\sigma \rho)>0$ of the total surplus is not extracted,

$$
E(T S \mid v)>\lim _{I \rightarrow \infty} E\left(R^{V} \mid v\right)=\lim _{I \rightarrow \infty} E\left(R^{U} \mid v\right)=\lim _{I \rightarrow \infty} E\left(R^{D} \mid v\right) .
$$

In the competitive limit, the residual supply of each bidder is perfectly elastic, and in the uniform-price auction, bidding becomes truthful, as in the Vickrey auction. With pay-as-bid pricing, by contrast, bidders still shade their marginal utility (see Equation (13)). Thus, the reasons underlying the seller's inability to extract surplus differ across the mechanisms: in the pay-as-bid auction, it is due to bid shading, whereas in the other two designs, it is attributed to the payment structure itself, which leaves part of surplus to the bidder. ${ }^{27}$

Less-than-full surplus extraction, even with symmetric information, is another instance of new effects brought by diminishing marginal utility that will hold outside of the setting analyzed here, in any equilibrium with downward-sloping bids. The less-than-full surplus extraction is also different from large auctions in single-unit demand settings where full surplus extraction, and hence revenue equivalence, has been established by the literature on information aggregation, cf. Pesendorfer and Swinkels (1997) and Kremer (2002). One insight from our simple setting with symmetric information is that the competitive-market result does not follow from the standard revenue equivalence theorem for unit demands. In all three auction formats, the allocation is efficient, while the revenue is strictly lower than the total surplus, which can be extracted (Footnote (25)).

With a finite number of bidders, a risk-averse seller faces a risk-revenue tradeoff when selecting an auction format. First, consider the competitive limit in which no such trade-off arises. By the equality of expected revenue in the competitive limit, a risk-neutral seller concerned about the expected revenue will be indifferent among the three mechanisms. Proposition 9 asserts that a risk-averse seller strictly prefers the uniform-price auction to the pay-asbid auction when there are sufficiently many bidders and the bidders' marginal utility is decreasing.

Proposition 9 (SOSD) For any strictly concave increasing utility function $\bar{u}(\cdot)$, in the unique linear equilibrium, there exists $\bar{I}$ such that for any $I \geq \bar{I}$,

$$
\mathbb{E}(\bar{u}(T S) \mid v)>\mathbb{E}\left(\bar{u}\left(R^{V}\right) \mid v\right)>\mathbb{E}\left(\bar{u}\left(R^{U}\right) \mid v\right)>E\left(\bar{u}\left(R^{D}\right) \mid v\right) .
$$

\footnotetext{
${ }^{27}$ In a study of large auctions, Swinkels (2001) obtained expected-revenue equivalence between the uniformprice and pay-as-bid auctions in an indivisible good, multi-unit demand setting with independent private values. Our model is a continuous-bid, complete information counterpart of his setting.
} 
In the limit,

$$
\lim _{I \rightarrow \infty} \mathbb{E}(\bar{u}(T S) \mid v)>\lim _{I \rightarrow \infty} \mathbb{E}\left(\bar{u}\left(R^{V}\right) \mid v\right)=\lim _{I \rightarrow \infty} \mathbb{E}\left(\bar{u}\left(R^{U}\right) \mid v\right)>\lim _{I \rightarrow \infty} E\left(\bar{u}\left(R^{D}\right) \mid v\right)
$$

Theorem 3 and Proposition 9 jointly define a trade-off faced by a seller when choosing an auction design in markets with strategic bidders. With sufficiently large $I$, the pay-as-bid auction gives higher expected revenue but also higher risk than the uniform-price auction. More generally, for any number of bidders, risk averse preferences exist for which either format is strictly preferred by the seller: For any $I$ sufficiently high, the c.d.f. of the revenue induced by the uniform-price auction crosses (once) the c.d.f. of the revenue in the pay-as-bid auction from below (as shown in the proof of Proposition 9). At the same time, Theorem 3 implies that the second-order stochastic dominance does not extend to auctions with a small number of bidders. Our results suggest that the uniform-price auction is more likely to be superior in markets with many bidders, whereas the pay-as-bid might be favored in small markets.

\section{$5.4 \quad$ Entry}

An important lesson from auction theory with single-unit demand is that a seller should favor auction formats that encourage greater participation (e.g., Bulow and Klemperer (1996)). With an additional bidder, other participants bid more aggressively. This recommendation is even more relevant in the context of divisible good auctions with decreasing marginal utility: apart from the competitive effect, additional participants increase the total surplus in the auction, even if bidders have identical marginal utilities. For quick intuition, consider a seller offering two units of a good to identical bidders with utility function $u(q)=2 q-0.5 q^{2}$. Allocating the two units to one bidder brings the total surplus of 2 , but allocating the good to two bidders so that each bidder receives one unit, increases the total surplus to 3 . If the identical demands were flat, the total surplus would be independent of the number of bidders.

Let us allow an infinite pool of potential entrants in the model. Having learned $v$, the bidders - simultaneously or sequentially - choose whether or not to join an auction. Entry incurs a fixed $\operatorname{cost} c$. Given the exogenous distribution of $Q$, per capita supply depends on the number of entrants. Consider pure strategy Subgame Perfect Nash equilibria such that the entrants submit linear bids in the bidding stage. Barring indifference, the number of entrants equals the maximal integer for which the expected bidder's payoff is greater than $c$.

Our main observation regarding entry is that, in any equilibrium, the uniform-price auction encourages weakly more entry than the Vickrey auction, which in turn encourages weakly more entry than the pay-as-bid auction; there exist values of parameters for which the inequalities are strict. In equilibrium, the uniform-price auction leaves more surplus 
to the bidders; given the fixed number and symmetry of the bidders, the allocations in all auction formats are Pareto efficient, and the total surplus is shared between the bidders and the seller. The ranking of entry then follows from the revenue rankings of Theorem 3 . The weak relative advantage of the uniform-price design in encouraging entry is consistent with the evidence from the U.S. Treasury experiment.

The entry advantage of the uniform-price auction turns out to be sufficient to reverse the revenue rankings established for auctions with a fixed number of participants. A small difference in the number of bidders translates into a significant revenue change, due to the surplus effect as well as the competitive effect. As a result, there are parameter values such that the uniform-price auction dominates the other auction formats in both expected revenue and participation. Thus, Friedman's conjecture about participation margin giving an advantage to the uniform-price auction holds even without information acquisition.

Ignoring the integer problem, one can show that the endogenized number of participants exceeds the Pareto efficient auction size in all three auction formats - an argument in favor of the pay-as-bid auction. In all auction formats, excess entry arises because each bidder ignores the negative externality of his participation on the net utility of other bidders. For auctions of single items, Levin and Smith (1994) demonstrate that auctions encourage entry levels that are excessive from a social point of view.

\section{Conclusions}

Multi-unit auctions differ from single-unit auctions in essential ways. Most fundamentally, the classic efficiency result for the second-price auction of a single item does not extend to the uniform-price auction of many items. In the uniform-price auction, winning bidders affect the market price with positive probability. Hence, bidders have incentives to reduce their demands, upsetting both the strategic simplicity and the efficiency of uniform-price auctions. By shading his bid for marginal items, the bidder is able to reduce the expected price paid on inframarginal items. The more one buys, the greater the incentive to shade. As a result, large bidders will sometimes lose against small bidders on items that the large bidders value more highly.

In this paper, we prove the general inefficiency of the uniform-price auction. Differential incentives to shade bids arise whenever a winner influences the market-clearing price with positive probability. The only cases that escape our inefficiency result are: (1) pure common values, in which all assignments are efficient, and (2) single-unit demands and analogous cases, where a bidder determines the price only when the bidder wins zero quantity.

An implication of the inefficiency result is that there is a class of environments (namely, symmetric private value auctions) in which the symmetric equilibrium of the oft-criticized pay-as-bid auction dominates all equilibria of the uniform-price auction in both efficiency and 
seller revenues. However, relaxing the symmetry and risk-neutrality assumptions leads to a class of environments where the uniform-price auction outperforms the pay-as-bid auction in both efficiency and revenues. Determining the better pricing rule is therefore an empirical question.

The practical importance of demand reduction is easily seen in spectrum auctions. Our theorems do not directly apply to the spectrum auctions, since the FCC and others used a simultaneous ascending auction and, often, the licenses are not perfect substitutes. Hence, the analogy between our setting and the spectrum auctions is crude. Still, based on the experience of many spectrum auctions around the world, we conclude that demand reduction is of fundamental importance to bidders. Indeed, demand reduction is likely more pronounced in simultaneous ascending auctions than in sealed-bid auctions, since the bidders can propose divisions of the licenses through their early bids. ${ }^{28}$ The October 1999 German auction of GSM spectrum, in which ten spectrum blocks were offered and the two principal bidders won five apiece, illustrates this behavior most clearly. The auction lasted just two rounds - one to propose the split and one to accept it.

Direct evidence of strategic demand reduction was observed in the FCC's Nationwide Narrowband Auction. In round 11, PageNet decided to cut back from bidding on three large licenses to two (Cramton 1995). PageNet felt that, if it continued to demand a third large license, it would drive up the prices on all of the large licenses to disadvantageously-high levels. Hence, it made sense to reduce its demand to two, even though the auction price had not yet reached PageNet's incremental value for a third large license. In making this decision, it was essential for PageNet to anticipate the effect of demand reduction on prices.

Anticipating price movements as a function of one's demand is often guesswork. Still the consequences of guessing wrong can be dramatic, as was illustrated in the August 2000 German auction of third-generation (3G) mobile wireless licenses. After round 127, Deutsche Telekom could have likely brought the auction to a rapid close by reducing its demand from three license blocks to two. Instead, Deutsche Telekom continued bidding for three blocks for some 40 more rounds, ultimately buying the two license blocks that it could have bought earlier, but paying about $\$ 2$ billion extra.

As in experiments, real-world bidders learn from their mistakes. Three months later, in November 2000, the Austrian 3G auction was held, with essentially the same rules and essentially the same players as the German auction. The starting prices in the Austrian auction were one-seventh of the final German prices (on a population-adjusted basis) and there were sufficiently few bidders that all could be winners if they reduced their demands. All but one of the bidders engaged in demand reduction at the first opportunity and there was a wide presumption that the one holdout, government-owned Telekom Austria, was under severe political pressure to prevent the auction from ending. Even then, demand reduction

\footnotetext{
${ }^{28}$ See, for example, Ausubel and Schwartz (1999).
} 
did well at predicting the outcome: the auction ended in just 14 rounds, at prices only $15 \%$ above the low starting prices, and with most participants shading their marginal bids well below their presumed values.

Another important application is in wholesale electricity markets. With only a few exceptions, these are uniform-price auctions conducted daily by the system operator. Our theory applies directly, accounting for the strategic supply reduction in response to uniform pricing in a procurement auction. The incentive to inflate bids grows with the quantity supplied, since the higher price is enjoyed on the larger quantity. Wolfram (1998) found compelling evidence of supply reduction in the early years of the UK electricity market. In 2001, the UK switched from uniform pricing to pay-as-bid pricing. The study by Borenstein, Bushnell, and Wolak (2002) of why electricity expenditures in California's restructured wholesale market rose from $\$ 2$ billion in summer 1999 to $\$ 9$ billion in summer 2000 found that over one-half of this increase was attributable to market power. In response to the crisis, the California Power Exchange considered switching from uniform to pay-as-bid pricing. Unlike in the UK, the proposal was rejected (Kahn et al. 2001).

We present this experience from electricity and spectrum markets to highlight the practical importance of demand reduction, rather than necessarily as an argument against the uniform-price auction. Uniform pricing has several desirable properties, including: (1) it is easily understood in both static and dynamic forms, (2) it is fair in the sense that the same price is paid by everyone, (3) absent market power it is efficient and strategically simple ("you just bid what you think it's worth"), and (4) the exercise of market power under uniform pricing favors smaller bidders. While the first three points are commonly made in practice, it is the fourth point that may decisively favor uniform pricing in many practical settings, including some spectrum and electricity markets.

Competition and innovation are often fostered by market designs that encourage the entry and success of small participants. Pay-as-bid pricing disadvantages small bidders: profits depend critically on the bidder's ability to guess the clearing price, and this ability grows with size. In sharp contrast, uniform pricing levels the playing field by weakening the penalty for guessing wrong. At the same time, the current paper has shown that uniform pricing also creates an incentive for large bidders to make room for their smaller rivals.

In his original proposal, Milton Friedman (1960, p. 65) recognized the informational leveling effect of uniform pricing: "This alternative, in any of its variants, will make the price the same for all purchasers, reduce the incentive for collusion, and greatly widen the market." We add to Friedman's effect the demand reduction effect, which cuts in the same direction. The Treasury auction experiment provides empirical support for the prediction that uniform pricing widens market participation: the five-firm concentration ratio declined by 10 percentage points in auctions that were changed from pay-as-bid to uniform pricing (Malvey and Archibald 1998). The uniform-price auction is not a panacea, since unlike its 
unit-demand counterpart it inevitably yields inefficiency, whereas some alternative multi-unit designs do not (see Vickrey (1961) and Ausubel (2004)). Nonetheless, good market design should encourage the evolution toward more competitive market structures, and uniform pricing does just that.

\section{References}

Armantier, O. And E. Sbai (2006): "Estimation and Comparison of Treasury Auction Format when Bidders are Asymmetric," Journal of Applied Econometrics, 21, 6, 745-79.

Ausubel, L. M. (2004): "An Efficient Ascending-Bid Auction for Multiple Objects," American Economic Review, 94, 5, 1452-75.

Ausubel, L. M. and P. Cramton (1999): "The Optimality of Being Efficient," Working Paper. Ausubel, L. M. And J. A. Schwartz (1999): "The Ascending Auction Paradox," Working Paper.

Back, K. And J. F. Zender (1993): "Auctions of Divisible Goods: On the Rationale for the Treasury Experiment," Review of Financial Studies, 6, 733-64.

Bolle, F. (1997): "Necessary Conditions for Efficient Multiple-Bid Auctions," in R. Nau, E. Gronn, M. Machina and O. Bergland, eds., Economic and Environmental Risk and Uncertainty: New Models and Methods, Boston: Kluwer Academic Publishers, 123-143.

Borenstein, S., J. B. Bushnell, And F. A. Wolak (2002): "Measuring Market Inefficiencies in California's Restructured Wholesale Electricity Market," American Economic Review, 92, 5, 1376-1405.

Brenner, M., D. Galai and O. Sade (2009): "Sovereign Debt Auctions: Uniform or Discriminatory?," Journal of Monetary Economics, 56, 267-274.

Bulow, J. I. And P. D. Klemperer (1996): "Auctions vs. Negotiations," American Economic Review, 86, 180-194.

Chapman, J. T. E., D. McAdams, and H. J. PaArsch (2007): "Bounding revenue comparisons across multi-unit auction formats under best response," American Economic Review, 97, 2, 455-458.

Cramton, P. (1995): "Money Out of Thin Air: The Nationwide Narrowband PCS Auction," Journal of Economics and Management Strategy, 4, 267-343.

Engelbrecht-Wiggans, R. (1988): "Revenue Equivalence in Multi-object Auctions," Economics Letters, 26, 15-19.

Engelbrecht-Wiggans, R., J. A. List, And D. Lucking-Reiley (2006): "Demand Reduction in Multi-unit Auctions with Varying Numbers of Bidders: Theory and Field Experiments," International Economic Review, 47, 1, 203-231.

Engelbrecht-Wiggans, R. And C. M. Kahn (1998): "Multi-Unit Auctions with Uniform Prices," Economic Theory, 12, 2, 227-258.

Federico, G. And D. Rahman (2003): "Bidding in an Electricity Pay-as-Bid Auction," Journal of Regulatory Economics, 24, 2, 175-211. 
FÉvrier, P., R. Préget And M., Visser (2004): "Econometrics of Share Auctions," Working Paper.

Friedman, M. (1960): A Program for Monetary Stability, New York, NY: Fordham University Press.

Glebkin S. And M. Rostek (2014): "Divisible Good Auctions: Designing Bidders' Valuation," Working Paper.

Hasto, P. And Holmberg, P. (2006): "Some Inequalities Related to Electricity Auctions," Applied Mathematics Letters, 19, 8, 814-819.

Holmberg, P. (2009): "Supply Function Equilibria of Pay-as-Bid Auctions," Journal of Regulatory Economics, 36, 154-177.

Hortaçsu, A. (2002): "Bidding Behavior in Divisible Good Auctions: Theory and Empirical Evidence from Turkish Treasury Auctions," Working Paper,.

HortaÇsu, A. And D. MCAdams (2010): "Mechanism Choice and Strategic Bidding in Divisible Good Auctions: An Empirical Analysis of the Turkish Treasury Auction Market," Journal of Political Economy , 188, 5, 833-865.

Hortaçsu, A. And S. Puller (2008): "Understanding Strategic Models of Bidding in Deregulated Electricity Markets: A Case Study of ERCOT ," RAND Journal of Economics, 39, 1, 86-114.

Jackson, M. O. And I. Kremer (2006): "The Relevance of a Choice of Auction Format in a Competitive Environment," Review of Economic Studies, 73, 4, 941-960.

Kagel, J. H. And D. Levin (2001): "Behavior in Multi-Unit Demand Auctions: Experiments with Uniform Price and Dynamic Vickrey Auctions," Econometrica, 69, 413-454.

Kahn, A. E., P. Cramton, R. H. Porter, and R. D. Tabors (2001): "Pricing in the California Power Exchange Electricity Market: Should California Switch from Uniform Pricing to Pay-as-Bid Pricing?" Blue Ribbon Panel Report, California Power Exchange.

Kastl, J. (2010): "Discrete Bids and Empirical Inference in Divisible Good Auctions," Review of Economic Studies, 78, 3, 974-1014.

Klemperer, P. D. AND M. A. Meyer (1989): "Supply Function Equilibria in Oligopoly under Uncertainty, " Econometrica, 57, 6, 1243-1277.

Kremer, I. (2002): "Information Aggregation in Common Value Auctions," Econometrica, 70, $1675-1682$.

Kyle, A. S. (1989): "Informed speculation with imperfect competition," Review of Economic Studies, 56, 317-56.

Levin, D. And J. L. Smith (1994): "Equilibrium in Auctions with Entry," American Economic Review, 84, 585-599.

List, J. A. And D. Lucking-Reiley (2000): "Demand Reduction in Multi-Unit Auctions: Evidence from a Sportcard Field Experiment," American Economic Review, 90:4, 961-972.

Malvey, P. F. And C. M. Archibald (1998): "Uniform-Price Auctions: Update of the Treasury Experience," U.S. Department of the Treasury, Washington D.C.,

http://www.treas.gov/offices/domestic-finance/debt-management.

Malvey, P. F., C. M. Archibald, and S. T. Flynn (1996): "Uniform-Price Auctions: Evalu- 
ation of the Treasury Experience," U.S. Department of the Treasury, Washington D.C., http://www.treas.gov/offices/domestic-finance/debt-management.

Maskin, E. And J. Riley (2000): "Asymmetric Auctions," Review of Economic Studies, 67, 413-438.

Milgrom, P. And R. J. Weber (1982): "A Theory of Auctions and Competitive Bidding," Econometrica, 50, 1089-1122.

NoussaIR, C. (1995): "Equilibria in a Multi-Object Uniform Price Sealed Bid Auction with MultiUnit Demands," Economic Theory, 5, 337-351.

Pycia, M. And K. Woodward (2014): "Pay As Bid: Selling Divisible Goods to Informed Bidders," Working Paper.

Rostek, M. And M. Weretka (2012): "Price Inference in Small Markets", Econometrica, 80, 687-711.

Pesendorfer, W. And J. Swinkels (1997): "The Loser's Curse and Information Aggregation in Common Value Auctions," Econometrica, 65, 1247-1281.

Smith, V. L.(1967): "Experimental Studies of Discrimination versus Competition in Sealed-Bid Auction Markets," Journal of Business, 40, 58-84.

Smith, V. L. (1982): "Microeconomic Systems as an Experimental Science," American Economic Review, 72, 923-955.

Swinkels, J. (2001): "Efficiency of Large Private Value Auctions," Econometrica, 61, 37-68.

Tenorio, R. (1997): "On Strategic Quantity Bidding in Multiple Unit Auctions," Journal of Industrial Economics, 95, 207-217.

Vickrey, W. (1961): "Counterspeculation, Auctions, and Competitive Sealed Tenders," Journal of Finance, 16, 8-37.

Vives, X. (2011): "Strategic Supply Function Competition with Private Information," Econometrica, 79, 1919-1966.

Wang J. J. D. And J. F. Zender (2002): “Auctioning Divisible Goods," Economic Theory, 19, 673-705.

Weretka, M. (2014): “A Note on Two-Unit Auctions with Diminishing Values," Working Paper.

Wilson, R. (1979): "Auctions of Shares," Quarterly Journal of Economics, 94, 675-689.

Wolak, F. (2003): "Identification and Estimation of Cost Functions Using Observed Bid Data: An Application to Electricity," Advances in Econometrics, 133-169.

Wolak, F. (2007): "Quantifying the Supply-Side Benefits from Forward Contracting in Wholesale Electricity Markets," Journal of Applied Econometrics, 22, 7, 1179-1209.

Wolfram, C, D. (1998): "Strategic Bidding in a Multi-Unit Auction: An Empirical Analysis of Bids to Supply Electricity in England and Wales," Rand Journal of Economics, 29, 703-725.

\section{Appendix A. Proofs}

Proof. Proposition 1 (EFficient BIDs): First, we demonstrate that, in an ex post efficient equilibrium, each bidder must use a flat-bid function, almost everywhere in types. Ex 
post efficiency and the simplifying assumptions we imposed on bidders' capacities $\lambda_{i}$ require that bidder $i$ wins $q_{i}=\lambda_{i}$ if bidder $i$ has the highest type and $q_{i}=0$ if bidder $i$ has the lowest type. Take any $s_{i}^{\prime}>s_{i}$. With positive probability all other bidders' types, $s_{-i}$, lie strictly between $s_{i}$ and $s_{i}^{\prime}$ and hence efficiency requires that $s_{i}^{\prime}$ must win $\lambda_{i}$ and $s_{i}$ must win 0 , and thus $b_{i}\left(\lambda_{i}, s_{i}^{\prime}\right) \geq b_{i}\left(0, s_{i}\right)$. Define $\phi_{i}\left(s_{i}\right)=\frac{1}{2}\left[b_{i}\left(0, s_{i}\right)+b_{i}\left(\lambda_{i}, s_{i}\right)\right]$; since bids are downward-sloping the preceding observation implies that $\phi_{i}(\cdot)$ is a weakly increasing function. Also define $S_{i}=\left\{s_{i} \in(0,1) \mid \phi_{i}(\cdot)\right.$ is differentiable at $\left.s_{i}\right\}$, and observe that that $b_{i}\left(0, s_{i}\right)=b_{i}\left(\lambda_{i}, s_{i}\right)$ for every $s_{i} \in S_{i}$. Furthermore, since a monotonic function is differentiable almost everywhere, the measure of $S_{i}$ equals one for all $i=1, \ldots, I$. Since $b_{i}\left(\cdot, s_{i}\right)$ is weakly decreasing in $q$, we conclude that $b_{i}\left(\cdot, s_{i}\right)$ is constant in $q$ for almost every type $s_{i}$.

Second, bidders use symmetric bid functions almost everywhere in types. Otherwise, there exist $i \neq j$ and $x \in S_{i} \cap S_{j}$ such that $\phi_{i}(x)<\phi_{j}(x)$. Using the continuity of $\phi_{i}(\cdot)$ and $\phi_{j}(\cdot)$ at $x$, there exist $s_{i} \in S_{i}$ and $s_{j} \in S_{j}$ such that $s_{i}>x>s_{j}$ but $\phi_{i}\left(s_{i}\right)<\phi_{j}\left(s_{j}\right)$. Then when all other bidders' signals, $s_{-i, j} \equiv\left\{s_{h}\right\}_{h \neq i, j}$, lie strictly between $s_{i}$ and $s_{j}$ (a positive-probability event), $s_{i}$ must win $\lambda_{i}$ and $s_{j}$ must win 0 , but this cannot happen if $b_{i}\left(\cdot, s_{i}\right)=\phi_{i}\left(s_{i}\right)<\phi_{j}\left(s_{j}\right)=b_{j}\left(\cdot, s_{j}\right)$. We conclude that $\phi_{i}(x)=\phi_{j}(x)$ for almost every type $x$, and we write $\phi(x)$ for the common bid.

Third, $\phi(\cdot)$ is strictly increasing. Otherwise, there exist $x^{\prime}>x$ such that $\phi_{i}(x)=\phi_{j}\left(x^{\prime}\right)$, and therefore $s_{i}, s_{i}^{\prime} \in S_{i}$ such that $x^{\prime}>s_{i}^{\prime}>s_{i}>x$ and $\phi\left(s_{i}^{\prime}\right)=\phi\left(s_{i}\right)$. We can then repeat the same argument as above: when all other bidders' types, $s_{-i}$, lie strictly between $s_{i}$ and $s_{i}^{\prime}$ (a positive-probability event), $s_{i}^{\prime}$ must win $\lambda_{i}$ and $s_{i}$ must win 0 for efficiency. But this cannot happen if $b_{i}\left(\cdot, s_{i}^{\prime}\right)=b_{i}\left(\cdot, s_{i}\right)$.

The claim for the uniform-price auction follows from the lemma below.

Lemma 2 Suppose that there exists an ex post efficient equilibrium of the uniform-price auction in a flat demands environment that exhibits the Generalized Winner's Curse. Then the expected value to bidder $i$ conditional on winning a small quantity is independent of $i$, that is, there exists $w^{+}(\cdot, \cdot)$ such that $w_{i}^{+}(x, x)=w^{+}(x, x)$, for every bidder $i=1, \ldots, I$ and every $x \in[0,1]$. Moreover, every bidder $i$ uses the symmetric flat-bid function $b_{i}\left(q, s_{i}\right)=$ $\phi\left(s_{i}\right)=w^{+}\left(s_{i}, s_{i}\right)$, for every type $s_{i} \in[0,1]$ and every quantity $q \in\left[0, \lambda_{i}\right]$.

Proof. For each $i=1, \ldots, I$, define

$$
J_{-i} \equiv \arg \max _{I^{\prime} \subset\{1, \ldots, I\} / i}\left\{\sum_{j \in I^{\prime}} \lambda_{j} \mid \sum_{j \in I^{\prime}} \lambda_{j}<1\right\} \text { and } \underline{L}_{i} \equiv 1-\sum_{j \in J_{-i}} \lambda_{j} .
$$

If there are multiple possible sets $J_{-i}$, select one arbitrarily. $J_{-i}$ is a combination of bidders other than bidder $i$ with a combined capacity $1-\underline{L}_{i}$ closest to, but strictly less than, one (the total quantity available). Note that $\underline{L}_{i}>0$; if in equation $(16), \underline{L}_{i}>\lambda_{i}$ 
redefine $\underline{L}_{i} \equiv \lambda_{i}$. By the proven above part of Proposition 1, in an efficient equilibrium, all bidders $j$ submit flat-bid schedules $b_{j}\left(q, s_{j}\right)=\phi\left(s_{j}\right)$ with probability one. Consider any bidder $i$, any quantity $q \in\left(0, \underline{L}_{i}\right)$, and almost any $s_{-i} \in(0,1)^{I-1}$. Then by equation (16), for any combination $J$ of opponents of bidder $i$, we have $q+\sum_{j \in J} \lambda_{j} \neq 1$ establishing that bidder $i$ 's bid is not pivotal. Note that $w_{i}^{q}(x, y)$ and $f_{i}^{q}(y \mid x)$ are constant in $q$ on the interval $\left(0, \underline{L}_{i}\right)$, for every $x, y \in(0,1)$; consequently, $w_{i}^{q}(x, y)=w_{i}^{+}(x, y) \equiv \lim _{q \downarrow 0} w_{i}^{q}(x, y)$ and $f_{i}^{q}(x \mid y)=f_{i}^{+}(x \mid y) \equiv \lim _{q \downarrow 0} f_{i}^{q}(x \mid y)$ for all $q \in\left(0, \underline{L}_{i}\right)$. Therefore, bidder $i$ 's optimal strategy for $q \in\left[0, \underline{L}_{i}\right)$ is to bid $b$ which maximizes

$$
\underline{L}_{i} \int_{0}^{\phi^{-1}(b)}\left[w_{i}^{+}(x, y)-\phi(y)\right] f_{i}^{+}(y \mid x) d y .
$$

Recall that $\phi(\cdot)$ from Proposition 1 is monotonic, and hence continuous almost everywhere. Consider any $x$ at which $\phi(\cdot)$ is continuous. Also recall that for any such $x, b_{i}(q, x)=\phi(x)$ for all quantities $q \in\left[0, \lambda_{i}\right]$. Next, observe that the integrand of equation (17) is independent of $b$ and, in fact, $b$ enters into the expression only through the upper limit, $\phi^{-1}(b)$, on the integral. Thus, if the bid $b=\phi(x)$ is optimal, it must be the case that the integrand, evaluated at $y=\phi^{-1}(b)=x$, equals zero. (Otherwise, since the integrand of equation (??) is continuous in $y$ when evaluated at $y=x$, there must exist $\varepsilon>0$ such that either the integrand is positive for all $y \in(x, x+\varepsilon)$ or the integrand is negative for all $y \in(x-\varepsilon, x)$; either of these conclusions would contradict the optimality of $b=\phi(x)$.) But then, $\phi(x)=w_{i}^{+}(x, x)$. Moreover, this conclusion holds for every bidder, so that $w_{i}^{+}\left(s_{i}, s_{i}\right)=w_{j}^{+}\left(s_{i}, s_{i}\right) \equiv w^{+}\left(s_{i}, s_{i}\right)$, for all bidders $i, j=1, \ldots, I$, and for almost every type $s_{i} \in[0,1]$. Finally, $\phi\left(s_{i}\right)=w_{i}^{+}\left(s_{i}, s_{i}\right)$ is strictly increasing in $s_{i}$, so $b_{i}\left(q, s_{i}\right)=\phi\left(s_{i}\right)=w^{+}\left(s_{i}, s_{i}\right)$ for every type $s_{i}$.

Proof. Theorem 1 (Inefficiency of Uniform-Price Auction): Notice that if $\lambda_{i}=\lambda$ and $1 / \lambda$ is an integer, then $w_{i}^{q}(x, x)$ is constant on $\left(0, \lambda_{1}\right]$ and equal to $w_{i}^{+}(x, x)$. With $w_{i}^{+}(x, x)=w^{+}(x, x)$ for all $i$, bids $b_{i}(q, v)=w^{+}(x, x)$ for all $i$ constitute a Bayesian-Nash equilibrium in which, in a uniform-price auction, items are assigned to the bidders with highest values with probability one.

We have already proven part of the converse statement in Lemma 2: in any ex-post efficient equilibrium $w_{i}^{+}(x, x)$ does not depend on the bidder $i$. It remains to be shown that efficiency implies that $\lambda_{i}=\lambda$ and $1 / \lambda$ is an integer. Let us rename the bidders so that $\lambda_{1} \geq \lambda_{2} \geq \ldots \geq \lambda_{I}$

By way of contradiction, suppose that there exists an ex post efficient equilibrium of the uniform-price auction, but that either $\lambda_{i}=\lambda$, where $1 / \lambda$ is not an integer, or $\lambda_{i} \neq \lambda_{j}$ for some $i \neq j$. By Lemma 2, all bidders $i=1, \ldots, I$ use the bid function $b_{i}(q, x)=\phi(x)=w^{+}(x, x)$, for all quantities $q \in\left[0, \lambda_{i}\right]$. Take $\underline{L}_{1}$ defined in equation (16). To obtain a contradiction in the remainder of the proof we construct $\bar{L}_{1} \in\left(\underline{L}_{1}, \lambda_{1}\right]$ such that it is not a best response for 
bidder 1 to bid $b_{1}(q, x)=\phi(x)=w^{+}(x, x)$, for any $q \in\left(\bar{L}_{1}, \lambda_{1}\right]$.

Let us begin by observing that interval $\left(\underline{L}_{1}, \lambda_{1}\right]$ is nonempty. If capacities are equal, $\lambda_{i}=\lambda$ for all $i$, then $\underline{L}_{1}=1-m \lambda$ where $m$ is the greatest integer such that $m \lambda<1$; since $1 / \lambda$ is not an integer we conclude that $\underline{L}_{1}<\lambda$. If capacities are not equal, and hence $\lambda_{1}>\lambda_{I}$, define $j^{\prime}=\max \left\{j \mid j \notin J_{-1}\right\}$ where $J_{-1}$ is defined in equation (16). Observe that $j^{\prime} \neq 1$, since $\sum_{k=2}^{I} \lambda_{k} \geq 1$. There are two cases: $\lambda_{j^{\prime}}<\lambda_{1}$ (Case I), and $\lambda_{j}^{\prime}=\lambda_{1}$ (Case II). In Case I, consider the set $J_{-1} \cup\left\{j^{\prime}\right\}$. By the definition of $\underline{L}_{1}$, we have $1-\underline{L}_{1}+\lambda_{j^{\prime}} \geq 1$. In Case II, observe that $j^{\prime} \neq I$, so $I \in J_{-1}$. Consider the set $\left\{j^{\prime}\right\} \cup J_{-1} \backslash\{I\}$. By the definition of $\underline{L}_{1}$, we have $1-\underline{L}_{1}+\lambda_{j^{\prime}}-\lambda_{I} \geq 1$. In each case, this implies that $\underline{L}_{1}<\lambda_{1}$, as desired.

Next, define

$$
J \equiv \arg \min _{I^{\prime} \subset\{2, \ldots, I\}}\left\{\sum_{j \in I^{\prime}} \lambda_{j} \mid \sum_{j \in I^{\prime}} \lambda_{j}>1-\lambda_{1}\right\} \text { and } \bar{L}_{1} \equiv 1-\sum_{j \in J} \lambda_{j} .
$$

(If there are multiple possible sets $J$, select one arbitrarily.) Since the previously-defined set $J_{-1}$ has the property that $\sum_{j \in J_{-1}} \lambda_{j}>1-\lambda_{1}$, it satisfies the strict inequality restriction in problem (18). It follows that $\sum_{j \in J} \lambda_{j} \leq \sum_{j \in J_{-1}} \lambda_{j}$, implying that $\underline{L}_{1} \leq \bar{L}_{1}<\lambda_{1}$. (For the case of $\lambda_{i}=\lambda$, where $1 / \lambda$ is not an integer, $\underline{L}_{1}=\bar{L}_{1}=1-m \lambda$.) Now, $\tau_{1}^{q}\left(s_{-1}\right), F_{1}^{q}(y \mid x)$, $f_{1}^{q}(y \mid x)$ and $w_{1}^{q}(y, x)$ are constant in $q$ for $q \in\left(\bar{L}_{1}, \lambda_{1}\right]$, and we write $\tau_{1}^{2}\left(s_{-1}\right), F_{1}^{2}(y \mid x)$, $f_{1}^{2}(y \mid x)$ and $w_{1}^{2}(y, x)$, for these values, respectively. Also, $\tau_{1}^{q}\left(s_{-1}\right), F_{1}^{q}(y \mid x), f_{1}^{q}(y \mid x)$ and $w_{1}^{q}(y, x)$ are constant in $q$ for $q \in\left(0, \bar{L}_{1}\right)$, and we write $\tau_{1}^{1}\left(s_{-1}\right), F_{1}^{1}(y \mid x), f_{1}^{1}(y \mid x)$ and $w_{1}^{1}(y, x)$, respectively, for these values. In terms of notation introduced in Section 4.1, $w_{1}^{1}(x, x)=w_{1}^{+}(x, x)$.

Given type $x \in(0,1]$, consider an alternative strategy for bidder 1 of bidding $\hat{b}_{i}\left(q, s_{i}\right)=$ $\phi(x)$ for $q \in\left[0, \bar{L}_{1}\right]$, and bidding $\hat{b}_{i}\left(q, s_{i}\right)=\beta \leq \phi(x)$, for $q \in\left(\bar{L}_{1}, \lambda_{1}\right]$. Let $\Pi_{1}(\beta)$ denote the expected payoff from this two-step strategy given that the other firms are bidding $b_{i}(q, \cdot)=$ $\phi(x)$. We show that $d \Pi_{1}(\beta) / d \beta$ evaluated at $\beta=\phi(x)$ is negative, which implies that bidder 1 can strictly improve his payoff by using the two-step bid function with $\beta<\phi(x)$, rather than bidding $\phi(x)$ for all $q \in\left[0, \lambda_{1}\right]$.

The three regions of bidder types to consider in calculating $\Pi_{1}(\beta)$ are as follows:

1. $\phi^{-1}(\beta)$ is greater than $\tau_{1}^{2}\left(s_{-1}\right)$. Then bidder 1 wins quantity $\lambda_{1}$, and $\tau_{1}^{2}\left(s_{-1}\right)$ determines the price. The contribution to the expected payoff is

$$
\lambda_{1} \int_{0}^{\phi^{-1}(\beta)}\left[w_{1}^{2}(x, y)-w_{1}^{+}(x, y)\right] f_{1}^{2}(y \mid x) d y .
$$

2. $\phi^{-1}(\beta)$ is between $\tau_{1}^{1}\left(s_{-1}\right)$ and $\tau_{1}^{2}\left(s_{-1}\right)$. Then bidder 1 wins quantity $\bar{L}_{1}$, and $\beta$ determines 
the price. The contribution to the expected payoff is

$$
\bar{L}_{1} \int_{0}^{\phi^{-1}(\beta)} \int_{\phi^{-1}(\beta)}^{1}\left[\mathbb{E}\left(v_{1} \mid s_{1}=x, \tau_{1}^{2}\left(s_{-1}\right)=y, \tau_{1}^{1}\left(s_{-1}\right)=z\right)-\beta\right] f_{1}^{2,1}(y, z \mid x) d y d z
$$

where $f_{1}^{2,1}(y, z \mid x)$ denotes the joint density of $\tau_{1}^{2}\left(s_{-1}\right)=y$ and $\tau_{1}^{1}\left(s_{-1}\right)=z$, conditional on $s_{1}=x$.

3. $\phi^{-1}(\beta)$ is less than $\tau_{1}^{1}\left(s_{-1}\right)$. Then, when $x$ is greater than $\tau_{1}^{1}\left(s_{-1}\right)$, bidder 1 wins quantity $\bar{L}_{1}$, and $\tau_{1}^{1}\left(s_{-1}\right)$ determines the price. The contribution to the expected payoff is

$$
\bar{L}_{1} \int_{\phi^{-1}(\beta)}^{x}\left[w_{1}^{1}(x, z)-w_{1}^{+}(z, z)\right] f_{1}^{1}(z \mid x) d z .
$$

$\Pi_{1}(\beta)$ is the sum of these three integrals. Taking the derivative of each with respect to $\beta$ when evaluated at $\beta=\phi(x)$ and combining and simplifying terms yields,

$\frac{d \Pi_{1}}{d \beta}=-\bar{L}_{1} \operatorname{Pr}\left\{\tau_{1}^{1}\left(s_{-1}\right)<x<\tau_{1}^{2}\left(s_{-1}\right)\right\}+\left(\lambda_{1}-\bar{L}_{1}\right) \phi^{-1^{\prime}}(\phi(x))\left[w_{1}^{2}(x, x)-w_{1}^{+}(x, x)\right] f_{1}^{2}(x \mid x)$

Observe that the first term of the right-hand side of equation (21) is strictly negative, while the second term is weakly negative (since $w_{1}^{2}(x, x) \leq w_{1}^{+}(x, x)$, from the Generalized Winner's Curse assumption). Hence, bidder $i=1$ strictly gains by bidding $\beta<\phi\left(s_{1}\right)$ for $q \in\left(\bar{L}_{1}, \lambda_{1}\right]$, yielding a contradiction.

Proof. Proposition 2 (Efficient PAy-As-Bid Auction): The argument in the text showed that a necessary condition for an ex post efficient equilibrium of the pay-as-bid auction is equation (7). If $v_{i}$ and $v_{j}$ are i.i.d. and $\lambda_{i}=\lambda_{j}$, then by equation $(6), Q_{i}(\cdot)=Q_{j}(\cdot)$ and thus $\phi_{i}(\cdot)=\phi_{j}(\cdot)=\phi(\cdot)$. Furthermore, $\phi(\cdot)$ is strictly monotone increasing, so every bidder using the same bid function, $\phi(\cdot)$, leads to an efficient allocation. Finally, note that every bidder using $\phi(\cdot)$ from $(7)$ constitutes a Bayesian-Nash equilibrium.

Proof. Proposition 3 (Inefficient Pay-AS-Bid Auction): Suppose there exist bidders $i$ and $j$ such that the associated distribution functions, $F_{i}(\cdot)$ and $F_{j}(\cdot)$, are not identical. As before, a necessary condition for an ex post efficient equilibrium is that bidder $i$ 's bid function be given by $\phi_{i}(\cdot)$, defined by the right-hand side of equation (7). At the same time, another necessary condition is that bidder $j$ 's bid function be given by $\phi_{j}(\cdot)$, defined by replacing $F_{(m)}^{-i}$ and $F_{(m+1)}^{-i}$ with $F_{(m)}^{-j}$ and $F_{(m+1)}^{-j}$ on the right-hand side of equation (7). For generic $F_{j} \neq F_{i}$, the implied $\phi_{j}(\cdot) \neq \phi_{i}(\cdot)$ on sets of positive measure, contrary to Proposition 1 . We conclude that there cannot exist any ex post efficient equilibrium. Similarly, if the capacities $\lambda_{i}$ are not all equal, then equation (7) again implies that, if $\lambda_{j} \neq \lambda_{i}$, then $\phi_{j} \neq \phi_{i}$ on sets of 
positive measure. Hence, there again cannot exist an ex post efficient equilibrium.

Proof. Proposition 4 (Dominance of Pay-AS-Bid Auction) In such an environment, that revenues are maximized by allocating items in descending order of their values, i.e., revenue maximization coincides with efficiency. Since the pay-as-bid auction inherits the symmetric equilibrium of the first-price auction for a single item, it attains full efficiency and consequently maximizes revenues proving the first part of the proposition. Now, if $1 / \lambda$ is not an integer, then Theorem 1 and Proposition 2 tell us that there is no efficient equilibrium of the uniform-price auction; and using the previous sentences, there is no equilibrium of the uniform-price auction that maximizes revenues. In particular, this means that the equilibrium of the pay-as-bid auction dominates all equilibria of the uniform-price auction with respect both to efficiency and revenues.

Proof. Proposition 5 (Dominance of Uniform-Price Auction): The marginal revenues are monotonic in values as $M R_{i}(v)=v-\frac{1-F_{i}(v)}{f_{i}(v)}=v-\frac{1-F(v)}{f(v)}$ for all $i=1, \ldots, I$. Thus, revenues are maximized by allocating the item to the bidder with the highest value. In the uniform-price auction bidding one's true value is the unique equilibrium in undominated strategies, and this equilibrium attains full efficiency and consequently maximizes revenues, proving the first part of the proposition.

To prove the second part of the proposition, suppose that two of the bidders have different distributions of values. We will have established that the equilibrium of the uniform-price auction dominates the equilibrium of the pay-as-bid auction, with respect to both efficiency and revenues, provided we can show that the equilibrium of the pay-as-bid auction is inefficient. This is demonstrated as follows. Consider the case of $I=2$ bidders; we may then assume that $\underline{v}_{1}<\underline{v}_{2}$. Suppose that the pay-as-bid auction has an efficient equilibrium in undominated strategies. For efficiency, bidder 2 must use a monotonic bidding strategy, and all types $v_{1}<\underline{v}_{2}$ of bidder 1 must win the auction with zero probability. It follows that, for any $\epsilon>0$, type $\underline{v}_{2}+\epsilon$ of bidder 2 must bid at least $\underline{v}_{2}-\epsilon$. Otherwise, types $v_{1} \in\left(\underline{v}_{2}-\frac{\epsilon}{2}, \underline{v}_{2}\right)$ of bidder 1 could profitably deviate by bidding flat $\underline{v}_{2}-\epsilon$. Let $p$ be the probability that bidder 1's type is less than $\frac{1}{2}\left(\underline{v}_{1}+\underline{v}_{2}\right)$. By bidding $\frac{1}{2}\left(\underline{v}_{1}+\underline{v}_{2}\right)$, every type of bidder 2 can assure himself a payoff of at least $\frac{1}{2}\left(\underline{v}_{2}-\underline{v}_{1}\right) p>0$. Consequently, for $\epsilon$ sufficiently small, type $\underline{v}_{2}+\epsilon$ of bidder 2 does not optimally bid at least $\underline{v}_{2}-\epsilon$, a contradiction. We conclude that there is no efficient equilibrium of the pay-as-bid auction. A similar argument can be made with more than two bidders.

Proof. Proposition 6 (Equilibrium in Uniform-Price Auction): Statistic $\mu_{i}$ and c.d.f. of $x, G_{i}(\cdot)$, contain all of the payoff-relevant information about bids $b_{j}(\cdot, v), j \neq i$. A linear equilibrium is obtained in three steps: (1) we find the best response to the residual 
supply function, taking $\mu_{i}$ and $G_{i}(\cdot)$ as given; (2) given the best response, we determine $\mu_{i}$; and finally, (3) we derive $G_{i}(\cdot)$ from $F(\cdot) \cdot{ }^{29}$ In the first step, we discretize the distribution of intercept $x$ by partitioning its support into a countable number of intervals of length $\Delta x$. The discrete realization of the supply function - which defines a state and is indexed by $s$ - originates at the midpoint of the corresponding interval. The probability of state $s, \pi^{s}$, is equal to the probability of the interval assigned by the distribution function $G_{i}(\cdot)$. Then, as the length of the interval goes to zero, $\Delta x \rightarrow 0$, the limit bid constitutes a best response to a continuously distributed residual supply.

For a bidder $i$ who faces a residual supply with slope $\mu_{i}$, consider a small deviation from equilibrium bid function around the bid-quantity pair $(b, q)$ observed in equilibrium given state $s$. The deviation increases the equilibrium quantity by $d q$ and the equilibrium price by $d p=\mu_{i} d q$ in state $s$; in no other state are the stop-out prices or equilibrium quantities altered. This translates into a marginal utility gain by $d q \times \pi^{s} \times(v-\rho q)$. The deviation increases the payment for state $s$ in two ways: (1) more units are purchased at price $b$, which yields the change in payments of $d q \times b$ and (2) the price increases for all units $q$, and hence the payment increases linearly by $q \times \mu_{i} d q$. At the optimum, marginal benefit and cost are equal, $d q \times \pi^{s} \times(v-\rho q)=d q \times \pi^{s} \times\left(b+\mu_{i} \times q\right)$. The Euler equation holds for any $\Delta x$ and, by the Maximum Theorem, in the limit as $\Delta x \rightarrow 0$. This gives bid function $b_{i}(q, v)=v-\left(\rho+\mu_{i}\right) q$; denote its slope by $\psi_{i}=\rho+\mu_{i}$. Since $\rho>0$ and $\mu_{i} \geq 0$ we conclude that $\psi_{i}>0$ and $b_{i}(\cdot, v)$ are invertible and the inverse bids are $b_{i}^{-1}(p, v)=\frac{v-p}{\psi_{i}}$. From the market clearing condition $q_{i}+\sum_{j \neq i} \frac{v-p}{\psi_{j}}=Q$, and hence the price impact of bidder $i$ can be expressed as $\mu_{i}=\frac{1}{\sum_{j \neq i} \frac{1}{\psi_{j}}}$, giving

$$
\psi_{i}=\frac{1}{\sum_{j \neq i} \frac{1}{\psi_{j}}}+\rho
$$

Thus, each $\psi_{i}$ equals $\frac{1}{I-1}$ times the harmonic mean of other agents' $\psi_{j}$, plus a constant. For $I>2$, the mapping from $\left(\psi_{i}\right)_{i=1, \ldots, I}$ to $\frac{1}{I-1}$ times the profile of harmonic means (plus a constant) is a contraction; therefore the above system of equations has a unique solution, $\psi_{i}=(I-1) \rho /(I-2)$. Substituting $\psi_{i}$ in bid functions gives bids in terms of primitive parameters. The resulting bid functions are in equilibrium, and the uniqueness of solution of the above system of equations implies that this equilibrium is unique in the class of linear equilibria.

Proof. Lemma 1 (Linearity of $h(\cdot)$ ): (Only if) Suppose $h(\cdot)$ is linear on the support,

\footnotetext{
${ }^{29}$ To shorten the analysis one could rely on the first-order condition derived by Wilson (1979) (see also Hortaçsu 2002). We provide the slightly longer proof for completeness, and because we think that the discretization-based approach is more intuitive and elementary.
} 
and hence there exist $h_{0}, h_{1} \in \mathbb{R}$ such that $h(q)=h_{0}+h_{1} q$. Since $h(\cdot)$ is well-defined, $g(\cdot)>0$ on the support, and hence $G(\cdot)$ is strictly increasing. Therefore, there exists a unique $q^{*} \in \mathbb{R}$ such that $G\left(q^{*}\right)=\frac{1}{2}$. For any $q$ in the support, $g(q)=\frac{1-G(q)}{h_{0}+h_{1} q}$. The right-hand side is continuous in the interior of the convex support as $h_{0}+h_{1} q>0$, and thus the differential equation gives a unique solution $G(\cdot)$ on the interior of the support, up to a constant. Thus there can exist at most one $G(\cdot)$ for which $h(q)=h_{0}+h_{1} q$ and the median is given by $q^{*}$. If $h_{1}=0$, the only solution is an exponential distribution; hence, it is within the class. For any $h_{1} \neq 0$, define $\xi \equiv h_{1}, \sigma \equiv \frac{h_{0}+h_{1} q^{*}}{2^{h_{1}}}$, and $\alpha \equiv\left(h_{0}+h_{1} q^{*} / 2^{h_{1}}-h_{0}\right) / h_{1}$. Since $h_{0}+h_{1} q^{*}>0$, we have $\sigma>0$. Therefore, parameters $\xi, \alpha \in \mathbb{R}$ and $\sigma \in \mathbb{R}_{++}$define a Generalized Pareto distribution. It is straightforward to verify that, with thusly defined parameters, the inverse hazard rate of the Generalized Pareto distribution $h_{0}+h_{1} q$ and its median is $q^{*}$. By the uniqueness argument, there can be at most one such distribution. Hence, the Generalized Pareto Distribution coincides with $G(\cdot)$.

(If) Given $F(Q \mid v)=1-\left(1+\xi \frac{Q-\alpha}{\sigma I}\right)^{-\frac{1}{\xi}}$, the c.d.f. of per capita supply is $q=Q / I$ is $G(q)=1-\left(1+\xi \frac{q-\alpha}{\sigma}\right)^{-\frac{1}{\xi}}$. Thus, $h(q)=\sigma-\alpha \xi+\xi q$ and hence $h(\cdot)$ is linear.

Proof. Proposition 7 (Equilibrium in PAy-AS-Bid Auction): Consider bidder $i$ in pay-as-bid auction, whose residual supply has slope $\mu_{i}$ and intercept $x$ with c.d.f. $G_{i}(\cdot)$ and the corresponding density $g_{i}(\cdot)$. With a discrete $x$, agent $i$ is bidding against a countable family of residual supplies, and the best response is a step function. ${ }^{30}$ Therefore, in deriving the best response for discrete $x$, we restrict attention to the class of step functions. Consider a local perturbation of a bid to a new step function around $(b, q)$, observed in state $s$. In state $s$, the perturbation increases the obtained quantity by $d q$ and the equilibrium price by $d p=\mu_{i} d q$ and does not affect either of the two variables in any other state. With the deviation increasing his quantity in state $s$, the marginal benefit is $d q \times \pi^{s} \times(v-\rho q)$. To find the cost of the deviation, observe that in state $s$, the payment increases because additional units purchased at price $b$ augment the payment by $d q \times b$ and, in addition, more aggressive bidding raises the price by $d p=\mu_{i} d q$. Since the payment for all units up to $q-\Delta q$ is determined by the upper part of the bid function, the increased price changes the payment for $\Delta q$ units. Consequently, the price impact effect is given by $\Delta q \times \mu_{i} d q$. In addition, aggressive bidding inflicts a negative externality on the payments in higher states. In all higher states, the payment increases by $\Delta q \times \mu_{i} d q$ and $\Delta p \times d q$. The total marginal cost associated with aggressive bidding amounts to $d q \times \pi^{s} \times\left(b+\mu_{i} \Delta q\right)+d q \times\left(\Delta p+\mu_{i} \Delta q\right) \times \sum_{k>s} \pi^{k}$. At the optimum, the marginal benefit balances the marginal cost. Alternatively, the Euler equation equalizes the net marginal benefit in state $s$ with the negative externality inflicted on the

\footnotetext{
${ }^{30} \mathrm{As}$ in the proof of Proposition 6, a shorter analysis would rely on the first-order condition derived by Wilson (1979) (see also Hortaçsu 2002). We provide the slightly longer proof for completeness, and because we think that the discretization-based approach is more intuitive and elementary.
} 
payments in all higher (but not lower) states

$$
\frac{\pi^{s}}{\Delta x} \frac{\Delta x}{\Delta q} \times\left(v-\rho q-b-\mu_{i} \Delta q\right)=\left(\frac{\Delta p}{\Delta q}+\mu_{i}\right) \times \sum_{k>s} \pi^{k}
$$

The Euler equation (22) gives a necessary optimality condition for a partition of the intercept into intervals of size $\Delta x$. Let $\Delta x \rightarrow 0$. The distribution $G_{i}(\cdot)$ is smooth, hence $\pi^{s} / \Delta x=$ $g_{i}(x)+o(1)$. By the Maximum Theorem on compact intervals, the best response converges uniformly to the unique linear best reply $b_{i}(\cdot, v)$. Thus, the ratio $\Delta p / \Delta q$ converges to the slope of the best response, $\Delta p / \Delta q=\partial b_{i}(\cdot, v) / \partial q+o(1)$, and the ratio $\Delta x / \Delta q$ converges to the negative of the slope of the affine function $x(q)$ that maps the equilibrium quantities into the intercepts, which is the relation observed in a linear equilibrium, $\Delta x / \Delta q=-\partial x(\cdot) / \partial q+o(1)$. The minus sign reflects the fact that the equilibrium relation $x(q)$ has a negative slope. Note that with an infinitely-fine grid, the within-state price impact effect disappears as $\Delta q \rightarrow 0$ and $\mu_{i} \Delta q=o(1)$. Substituting the limits into (22), ignoring the $o(1)$ elements, and observing that the probability of all higher states coincides with $G_{i}(\cdot)$, gives the limit Euler equation

$$
-\frac{\partial x(\cdot)}{\partial q}[v-\rho q-b]=\left[\mu_{i}-\frac{\partial b_{i}(\cdot)}{\partial q}\right] \frac{G_{i}(x)}{g_{i}(x)} .
$$

We re-cast the Euler equation in terms of the distribution of the equilibrium quantity $q$. In equilibrium, $x(q)$ is an affine decreasing transformation of $q$, the c.d.f. of $q$ can be found as $G(q)=1-G_{i}(x(q))$ and the density of $q$ as $g(q)=-g_{i}(x) \times \partial x(\cdot) / \partial q$. The inverse hazard rate of the equilibrium quantity is defined by $h(q) \equiv(1-G(q)) / g(q)$. Thus, $v-\rho q-p=$ $h(q)\left[\mu_{i}-\partial b_{i}(\cdot) / \partial q\right] .^{31}$

Substituting in for $h(q)=\sigma+\xi q$ this last condition can be rewritten as

$$
v-\rho q-b=(\sigma+\xi q)\left(\mu_{i}-\psi_{i}\right)
$$

In a linear equilibrium, the slope of the bid function $\psi_{i} \equiv \partial b_{i}(\cdot) / \partial q$ is a constant. Solving for $b$, differentiating with respect to $q$, and solving for the slope of the bid gives $\psi_{i}=$ $\xi \mu_{i} /(1-\xi)+\rho /(1-\xi)$. Since $\rho>0$ and $\xi<\frac{I-1}{I}<1$ we conclude that $\psi_{i}>0$ and the bid functions are invertible, with the slope of inverse bids $\psi_{i}^{-1}=\frac{1}{\xi \mu_{i} /(1-\xi)+\rho /(1-\xi)}$. From the

\footnotetext{
${ }^{31}$ In the derivation of the first-order condition, we assume that the considered $q$ is in the support of the equilibrium quantity. When density $g(q)$ is equal to zero, inverse hazard rate is not well defined, and the first-order condition does not apply. If $q$ is smaller than the quantities in the support, a bidder has an incentive to submit the smallest possible bid. Aggressive bidding for such $q$ brings no benefit of greater quantity, while it does increase the payments in higher states. Given that submitted bids are required to be non-increasing, the optimal bid has flat parts. Note that the flat-bid parts do not occur for the quantities to the right of the support. For such quantities, the submitted bids have and effect neither on the equilibrium quantities nor on the payment in any of the possible states, and bidders are indifferent to what they submit.
} 
market clearing condition, $q_{i}-p \sum_{j \neq i} \psi_{i}^{-1}-Q$ is a constant, and hence the price impact of bidder $i$ can be expressed as $\mu_{i}=\frac{1}{\sum_{j \neq i} \psi_{j}^{-1}}$, giving

$$
\psi_{i}=\xi \mu_{i} /(1-\xi)+\rho /(1-\xi)=\frac{\xi}{1-\xi} \frac{1}{\sum_{j \neq i} \frac{1}{\psi_{j}}}+\text { constant }
$$

Thus each $\psi_{i}$ equals $\frac{\xi}{1-\xi} \frac{1}{I-1}$ times the harmonic mean of other agents' $\psi_{j}$, plus a constant. Notice that for $\xi<0$ we have $\left|\frac{\xi}{1-\xi} \frac{1}{I-1}\right|<1$; this bound remains true for $\xi \geq 0$ as long as $\xi<\frac{I-1}{I}$. We conclude that the mapping from $\left(\psi_{i}\right)_{i=1, \ldots, I}$ to $\frac{\xi}{1-\xi} \frac{1}{I-1}$ times the profile of harmonic means (plus a constant) is a contraction; therefore the above system of equations has a unique solution, $\psi_{i}=\frac{(I-1) \rho}{I(1-\xi)-1}$. The resulting bid functions are in equilibrium, and the uniqueness of solution of the above system of equations implies that this equilibrium is unique in the class of linear equilibria.

Proof. Theorem 3 (Expected Revenue Ranking): Given equilibrium bids, revenue per capita in three auction formats are

$$
\begin{aligned}
R^{U} & =v q-\frac{I-1}{I-2} \rho q^{2} \\
R^{D} & =\left(v-\frac{I \rho \sigma}{I-1-I \xi}\right) q-\frac{1}{2} \frac{(I-1) \rho}{I(1-\xi)} q^{2}, \\
R^{V} & =v q-\frac{2 I-1}{2 I-2} \rho q^{2},
\end{aligned}
$$

where $q=Q / I$. The total surplus per capita is given by

$$
T S=v q-\frac{1}{2} \rho q^{2}
$$

The first two moments of $q$ with Generalized Pareto distribution with $\alpha=0, \sigma>0$ and $\xi<1 / 2$ are given by $\mathbb{E}(q)=\frac{\sigma}{1-\xi}$ and $\mathbb{E}\left(q^{2}\right)=\frac{2 \sigma^{2}}{(1-\xi)(1-2 \xi)}$. The interim expected revenues are

$$
\begin{aligned}
\mathbb{E}\left(R^{U} \mid v\right) & =\frac{\sigma}{1-\xi}\left[v-\frac{I-1}{I-2} \frac{2 \rho \sigma}{(1-2 \xi)}\right], \\
\mathbb{E}\left(R^{D} \mid v\right) & =\frac{\sigma}{1-\xi}\left[v-\frac{I \rho \sigma}{I-1-I \xi}-\frac{I-1}{I-1-I \xi} \frac{\rho \sigma}{(1-2 \xi)}\right], \\
\mathbb{E}\left(R^{V} \mid v\right) & =\frac{\sigma}{1-\xi}\left[v-\frac{2 I-1}{I-1} \frac{\rho \sigma}{(1-2 \xi)}\right] .
\end{aligned}
$$

For all $I>2, \rho>0$, and $\xi<0$ strict inequalities $T S>\mathbb{E}\left(R^{D} \mid v\right)>\mathbb{E}\left(R^{V} \mid v\right)>\mathbb{E}\left(R^{U} \mid v\right)$ 
hold, as required. ${ }^{32}$

Proof. Proposition 8 (Ex Post Revenue Ranking): For any realization of $Q$ and $v$, the per-capita total surplus and the seller's revenues in the Vickrey auction and the uniform price auction are given by (24), (26), and (27), respectively. The revenue rankings follow.

Proof. Proposition 9 (SOSD): In light of Proposition 8 and the discussion preceding Proposition 9, it is enough to prove that in the limit as $I \rightarrow \infty$ and $q=Q / I$ stays constant, the expected seller's payoff is higher in the uniform-price auction than in the pay-as-bid auction. Let us fix $v$ and notice that for any realization of $q$, the revenue in the competitive limit of uniform-price and Vickrey auctions is given by $R^{U}=R^{V}=v q-\rho q^{2}$, whereas in a pay-as-bid auction, it is $R^{D}=\left(v-\frac{\rho \sigma}{1-\xi}\right) q-\frac{1}{2} \frac{\rho}{1-\xi} q^{2}$. Crucially, our non-satiation assumption implies that both $R^{U}=R^{V}$ and $R^{D}$ are strictly increasing in $q$ that belongs to the support of $Q / I$.

As the difference $R^{U}(q)-R^{D}(q)=\frac{\rho \sigma}{1-\xi} q-\frac{1-2 \xi}{2-2 \xi} \rho q^{2}$ is quadratic in $q$ and $R^{U}(0)=R^{D}(0)=$ 0 , there is at most one $q^{*}>0$ in the support of both random variables, for which $R^{*} \equiv$ $R^{U}\left(q^{*}\right)=R^{D}\left(q^{*}\right)$. Since, the revenues are equal in expectation over $q$, threshold $q^{*}$ exists and belongs to the interior of the support of per-capita supply. Because the revenue function in the uniform-price and the Vickrey auction is steeper at $q=0$ than that of the pay-as-bid, we conclude that $R^{U}(q)>R^{D}(q)$ for $q \in\left(0, q^{*}\right)$ and $R^{U}(q)<R^{D}(q)$ for $q>q^{*}$. Thus, with fixed $v$, in the interval $\left(0, R^{*}\right)$ the c.d.f. of the pay-as-bid auction revenue is strictly higher than that of the uniform-price auction, and for all $R>R^{*}$ the c.d.f. of the uniform-price auction revenue is greater than that of the pay-as-bid auction. Since the expected revenues are equal, this single-crossing of the two c.d.f.'s at $R^{*}$ is sufficient for the uniform-price auction revenue to stochastically dominate the pay-as-bid auction in the second-order sense. Continuity of revenues gives the assertion.

\section{Appendix B. Additional Examples}

\section{(Intended for Online Appendix)}

We have said little about existence and uniqueness of equilibria, nor about how one might go about constructing equilibria. In general, all of these remaining tasks may be problematic. In particular, we know from Wilson (1979) and subsequent papers that when the items are

\footnotetext{
${ }^{32}$ While our non-satiation assumption implies that $F$ has bounded support, and hence $\xi<0$, note that if $\xi \in(0,1 / 2)$ then our characterization of equilibria remains true, and $T S>\mathbb{E}\left(R^{V} \mid v\right)>\mathbb{E}\left(R^{D} \mid v\right)>\mathbb{E}\left(R^{U} \mid v\right)$ for all $v$.
} 
infinitely divisible, a vast multiplicity of equilibria is probably inherent to the uniform-price auction and, in any case, the calculation of equilibria may be difficult.

In this appendix, we give some examples where the above difficulties disappear. They generalize the examples of Section 2. These examples - which have simple theoretical outcomesare especially useful for experiments and classroom exercises. They are also useful in getting a sense of the theoretical magnitudes of the necessary efficiency losses - and possible revenue losses - inherent in the uniform-price auction.

Example B1. Only One Multi-Unit Bidder. Consider a uniform-price auction with $n+1$ bidders and $m$ indivisible identical items, where $n \geq m$. Bidders $1, \ldots, n$ possess positive marginal values only for a single unit, but Bidder 0 possesses positive marginal values for two or more units. This is a simple extension of the unit-demand model (Weber 1983). As in that model, a single round of elimination of weakly dominated strategies has substantial cutting power. Each bidder 1,.., nfinds that his bid cannot be pivotal in any state of the world in which he wins a unit, and so he bids his true value. After this round of elimination, Bidder 0 faces a simple decision problem whose solution, by Theorem 1 , inevitably involves demand reduction.

Further suppose bidder 0 places the same marginal value, $v_{0}$, on each unit, where $v_{0}$ is drawn from distribution function $F_{0}$. Each bidder $i=1, \ldots, n$ demands only one unit of the good, placing a marginal value of $v_{i}$ on the single unit, where each $v_{i}$ is independently drawn from distribution function $F$. The distribution functions $F_{0}$ and $F$ have the same support.

Let $v(k)$ denote the $k^{\text {th }}$-order statistic of $v_{1}, \ldots, v_{n}$ (the $k^{\text {th }}$-highest value excluding bidder $0)$. Bidder 0 performs a calculation analogous to that in the proof of Theorem 1 . On his first unit of quantity, bidder 0 bids $v_{0}$. Let $b$ denote his bid on the second unit, and let $\pi_{0}\left(v_{0}, b\right)$ denote his expected payoff from bidding $b$ when his true value is $v_{0}$. Then

$\pi_{0}\left(v_{0}, b\right)=2 \int_{0}^{b}\left(v_{0}-b\right) d F_{(m-1)}(p)+\left[v_{0}-b\right]\left[F_{(m)}(b)-F_{(m-1)}(b)\right]+\int_{b}^{v_{0}}\left(v_{0}-p\right) d F_{(m)}(p)$.

Differentiating with respect to $b$ and canceling terms yields the first-order condition

$$
\left[v_{0}-b\right] f_{(m-1)}(b)=F_{(m)}(b)-F_{(m-1)}(b) .
$$

Recognizing that $F_{(m)}(b)-F_{(m-1)}(b)=\left(\begin{array}{c}n \\ m-1\end{array}\right)[1-F(b)]^{m-1}[F(b)]^{n-m+1}$, we conclude

$$
b+\left(\frac{1}{m-1}\right)\left(\frac{1-F(b)}{f(b)}\right)=v_{0} .
$$

If this equation yields a unique $b$ for each realization $v_{0}$, then the model in which Bidder 0 pos- 
sesses positive marginal values for two units has a unique equilibrium in weakly-undominated strategies. When the implied $b$ is nonnegative, this gives Bidder 0's bid; when the implied $b$ is negative, Bidder 0 bids zero for the second unit.

Example B2. Only One Multi-Unit Bidder and Uniform Distributions. Continue Example 1, assuming $F(b)=b$. Equation (31) then yields

$$
b\left(v_{0}\right)=\left\{\begin{array}{cc}
\frac{(m-1) v_{0}-1}{m-2}, & \text { if } v_{0}>\frac{1}{m-1} \\
0, & \text { otherwise }
\end{array}\right.
$$

Example B3. All Bidders Desire Two Units, Supply Equals Two Units, and Uniform Distributions. First continue Example B2 by assuming that the supply equals two units $(m=2)$. Observe that Equation (32) implies that if one multi-unit bidder, who has positive marginal value for two units, bids against $n$ bidders, who each demand only a single unit, then $b\left(v_{0}\right)=0$. Thus, the two-unit bidder behaves in equilibrium as if he has a positive marginal value for only a single unit, and he bids his true value on the single unit.

Now suppose instead that each of the bidders desires two units. If all other bidders bid their true value on the first unit but zero on the second unit, the best response for the remaining bidder is also to bid his true value on the first unit but zero on the second unit. Thus, one equilibrium of the uniform-price auction is for every bidder to bid his true value on the first unit but to bid $b=0$ on the second unit. (Equilibria with this structure were discovered by Noussair (1995) and Engelbrecht-Wiggans and Kahn (1998), in models closely related to Example B3.)

This example is particularly striking in that it admits another equilibrium. Observe that it can be reinterpreted as a flat-demands model with $\lambda=1$ for all bidders. Consequently, it is also an equilibrium for every bidder to bid his true value on the first unit and to also bid his true value on the second unit. That is to say, Example B3 lies within one of the exceptions to the Inefficiency Theorem 1, but it nevertheless possesses a grossly inefficient equilibrium as well as a fully efficient equilibrium.

\section{Appendix C. Full Treatment of Example V}

\section{(Intended for Online Appendix)}

We now demonstrate the existence and the uniqueness of symmetric monotone equilibrium in the three auction formats.

Uniform-price auction: Existence: By the arguments analogous to those for flat demands (Example I), bidding strategies $b^{1}\left(v_{i}\right)=v_{i}$ and $b^{2}\left(v_{i}\right)=0$ for agents $i=1,2$ with de- 
creasing marginal utilities constitute an equilibrium. Relative to flat demands, assumption $\rho<1$ reduces incentives to bid for the second unit. Uniqueness: We argue by contradiction that with decreasing marginal utility, $\rho \in(0,1)$, the zero-revenue equilibrium is the only equilibrium within the class of symmetric, (weakly) monotone equilibria with continuously differentiable bids $\left(b^{1}(\cdot), b^{2}(\cdot)\right)$. Since the bid for the first unit never determines the price, in a symmetric equilibrium, we have $b^{1}\left(v_{i}\right)=v_{i}$, for otherwise the bidders could strictly increase their utilities. Now, suppose that $b^{2}(\cdot) \neq 0$ in equilibrium. It follows that there exists $v_{i}^{\prime}$ such that $b^{2}\left(v_{i}^{\prime}\right)>0$. Since $b^{2}(0)=0, v^{\prime} \neq 0$ and by the intermediate value theorem, there exists $v^{\prime \prime} \in\left(0, v^{\prime}\right)$, for which the slope of the bid function is strictly increasing, $\left[b^{2}\left(v^{\prime \prime}\right)\right]^{\prime}=\frac{b^{2}\left(v^{\prime}\right)-b^{2}(0)}{v^{\prime}}>0$. Since $b^{2}(\cdot)$ is $C^{1}$, there exists $\epsilon>0$ and a corresponding neighborhood $V=\left[v^{\prime \prime}-\epsilon, v^{\prime \prime}+\epsilon\right]$ on which $b^{2}(\cdot)$ is strictly positive and strictly increasing. Define $B=b^{2}(V)$ as an image of interval $V$. By the standard arguments, $B$ is an interval too. Restriction $b^{2}: V \rightarrow B$ is a bijection, with a well defined inverse $\phi^{2}: B \rightarrow V$. Moreover, $\phi^{2}(\cdot)$ is strictly increasing and differentiable. Given the equilibrium strategy for bidder $j$, for any $b \in B, \operatorname{Pr}\left[b^{2}\left(v_{j}\right) \leq b\right]=F\left[\phi^{2}(b)\right]=\frac{\phi^{2}(b)}{100}$ with density $\frac{\left[\phi^{2}(b)\right]^{\prime}}{100}$.

In the uniform-price auction, there are three events in which bidder $i$ wins: when $b_{i}^{2} \geq$ $b^{1}\left(v_{j}\right)$ in which case he wins two units and pays $v_{j}$; when $b^{1}\left(v_{j}\right) \geq b_{1}^{2} \geq b^{2}\left(v_{j}\right)$ in which case he wins one unit and pays $b_{1}^{2}$, and finally when $b_{1}^{1} \geq b^{2}\left(v_{j}\right)$ and $b^{2}\left(v_{j}\right) \geq b_{1}^{2}$. The expected payoff is then given by

$$
\begin{aligned}
\pi\left(b_{i}^{1}, b_{i}^{2}\right)= & F\left(b_{i}^{2}\right)(1+\rho) v_{i}-2 \int_{0}^{\phi^{1}\left(b_{i}^{2}\right)} v_{j} f\left(v_{j}\right) d v_{j} \\
& +\left[F\left(\phi^{2}\left(b_{i}^{2}\right)\right)-F\left(\phi^{1}\left(b_{i}^{2}\right)\right)\right]\left(v_{i}-b_{i}^{2}\right) \\
& +\left[F\left(\phi^{2}\left(b_{i}^{1}\right)\right)-F\left(\phi^{2}\left(b_{i}^{2}\right)\right)\right] v_{i}-\int_{\phi^{2}\left(b_{i}^{2}\right)}^{\phi^{2}\left(b_{i}^{1}\right)} b^{2}\left(v_{j}\right) f\left(v_{j}\right) d v_{j},
\end{aligned}
$$

which can be rewritten as

$$
\begin{aligned}
\pi\left(b_{i}^{1}, b_{i}^{2}\right)= & F\left(\phi^{1}\left(b_{i}^{2}\right)\right)(1+\rho) v_{i}-2 \int_{0}^{\phi^{1}\left(b_{i}^{2}\right)} v_{j} f\left(v_{j}\right) d v_{j} \\
& +\left[F\left(\phi^{2}\left(b_{i}^{1}\right)\right)-F\left(\phi^{1}\left(b_{i}^{2}\right)\right)\right] v_{i} \\
& -\left[F\left(\phi^{2}\left(b_{i}^{2}\right)\right)-F\left(\phi^{1}\left(b_{i}^{2}\right)\right)\right] b_{i}^{2}-\int_{\phi^{2}\left(b_{i}^{2}\right)}^{\phi^{2}\left(b_{i}^{1}\right)} b^{2}\left(v_{j}\right) f\left(v_{j}\right) d v_{j} .
\end{aligned}
$$

Using the assumption of the uniform distribution and truthful bidding for the first unit by agent $i$,

$$
\pi=b_{i}^{2} \rho v_{i}-\phi^{2}\left(b_{i}^{2}\right) b_{i}^{2}-\int_{\phi^{2}\left(b_{i}^{2}\right)}^{\phi^{2}\left(b_{i}^{1}\right)} b^{2}\left(v_{j}\right) f\left(v_{j}\right) d v_{j}+\phi^{2}\left(b_{i}^{1}\right) v_{i} .
$$


The first-order necessary condition implies

$$
\frac{\partial \pi}{\partial b_{i}^{2}}==\rho v_{i}-\phi^{2 \prime}\left(b_{i}^{2}\right) b_{i}^{2}-\phi^{2}\left(b_{i}^{2}\right)+b_{i}^{2} \phi^{2 \prime}\left(b_{i}^{2}\right)=\rho v_{i}-\phi^{2}\left(b_{i}^{2}\right)
$$

It follows that for $v_{i} \in V$, bidding strategy $b_{i}^{2}=b^{2}\left(v_{i}\right)$ gives a strictly negative value $\frac{\partial \pi}{\partial b_{i}^{2}}=$ $(\rho-1) v_{i}<0$ and, hence, given $v_{i}$, bidding $v_{i}, b^{2}\left(v_{i}\right)$ is not a best response to $b^{1}(\cdot), b^{2}(\cdot)$, a contradiction. It follows that, in any equilibrium, $b^{2}\left(v_{i}\right)=0$ and the zero revenue equilibrium is unique within the class of weakly monotone symmetric equilibria.

Pay-as-bid auction: Existence: We first derive a symmetric equilibrium in pay-as-bid auction in EXAMPLE 5: As shown in the text, the first order necessary and sufficient conditions give two differential equations

$$
\left[\phi^{1}(b)\right]^{\prime}=\frac{\phi^{1}(b)}{\rho \phi^{2}(b)-b},\left[\phi^{2}(b)\right]^{\prime}=\frac{\phi^{2}(b)}{\phi^{1}(b)-b} .
$$

Rearranging terms in (33) gives

$$
\left[\left[\phi^{1}(b)\right]^{\prime}-1\right]\left[\rho \phi^{2}(b)-b\right]=\phi^{1}(b)-\left[\rho \phi^{2}(b)-b\right]
$$

and

$$
\left[\rho\left[\phi^{2}(b)\right]^{\prime}-1\right]\left[\phi^{1}(b)-b\right]=\rho \phi^{2}(b)-\left[\phi^{1}(b)-b\right] .
$$

Adding both equations

$$
\left[\left[\phi^{1}(b)\right]^{\prime}-1\right]\left[\rho[\phi(b)-b]+\left[\rho\left[\phi^{2}(b)\right]^{\prime}-1\right][\phi(b)-b]=2 b,\right.
$$

which can be reduced to

$$
\frac{d}{d b}\left[\left[\rho \phi^{2}(b)-b\right]\left[\phi^{1}(b)-b\right]\right]=2 b .
$$

Integrating both sides and using the fact that $\phi^{1}(0)=\phi^{2}(0)=0$ gives

$$
\left[\rho \phi^{2}(b)-b\right]\left[\phi^{1}(b)-b\right]=b^{2} .
$$

Since $\phi^{1}(\bar{b})=\phi^{2}(\bar{b})=100$, it follows that $[100 \rho-\bar{b}][100-\bar{b}]=\bar{b}^{2}$, and hence,

$$
\bar{b}=\frac{100 \rho}{1+\rho}
$$


The first order conditions (33) combined with (34) give two independent differential equations

$$
\begin{aligned}
{\left[\phi^{1}(b)\right]^{\prime} } & =\frac{\phi^{1}(b)\left[\phi^{1}(b)-b\right]}{b^{2}}, \\
{\left[\phi^{2}(b)\right]^{\prime} } & =\frac{\phi^{2}(b)\left[\rho \phi^{2}(b)-b\right]}{b^{2}},
\end{aligned}
$$

one for each inverse bid function. It is straightforward to verify that the two differential equations have the following solutions

$$
\phi^{1}(b)=\frac{2 b}{1+c_{1} b^{2}},
$$

and

$$
\phi^{2}(b)=\frac{2 b}{\rho+c_{2} b^{2}} .
$$

The integration constants can be obtained from conditions $100=\phi^{k}(\bar{b})$, i. e. $c_{1}=\frac{\rho^{2}-1}{(100 \rho)^{2}}$ and $c_{2}=\frac{1-\rho^{2}}{(100)^{2} \rho}$. The bids can be obtained in closed from by inverting functions $\phi^{k}$ :

$$
\begin{aligned}
v_{i} & =\frac{2 b}{1+c_{1} b^{2}} \rightarrow v_{i} c_{1} b^{2}-2 b+v_{i}=0 \\
b^{1}\left(v_{i}\right) & =\frac{1}{v_{i} c_{1}}\left(1-\sqrt{1-c_{1}\left(v_{i}\right)^{2}}\right) \\
& =\frac{100^{2} \rho^{2}}{\rho^{2}-1} \frac{1}{v_{i}}\left(1-\sqrt{1-\frac{\rho^{2}-1}{100^{2} \rho^{2}}\left(v_{i}\right)^{2}}\right),
\end{aligned}
$$

and for the second bid,

$$
\begin{aligned}
v_{i} & =\frac{2 b}{\rho+c_{2} b^{2}} \rightarrow v_{i} c_{2} b^{2}-2 b+v_{i} \rho=0 \\
b^{2}\left(v_{i}\right) & =\frac{1}{v_{i} c_{2}}\left(1-\sqrt{1-c_{2} \rho\left(v_{i}\right)^{2}}\right)= \\
& =\frac{100^{2} \rho}{1-\rho^{2}} \frac{1}{v_{i}}\left(1-\sqrt{1-\frac{1-\rho^{2}}{100^{2}}\left(v_{i}\right)^{2}}\right) .
\end{aligned}
$$

We conclude by arguing that constraint $b^{1} \geq b^{2}$ is satisfied in equilibrium for all $v_{i}$. For $v_{i}=0$, bids are zero as well and, hence, the condition is not binding. For $v_{i}>0$, inequality 
$b^{1} \geq b^{2}$ holds if

$$
\begin{aligned}
& \frac{100^{2} \rho^{2}}{\rho^{2}-1} \frac{1}{v_{i}}\left(1-\sqrt{1-\frac{\rho^{2}-1}{100^{2} \rho^{2}}\left(v_{i}\right)^{2}}\right) \geq \frac{100^{2} \rho}{1-\rho^{2}} \frac{1}{v_{i}}\left(1-\sqrt{1-\frac{1-\rho^{2}}{100^{2}}\left(v_{i}\right)^{2}}\right) \\
& -\rho+\sqrt{\rho^{2}-\frac{\rho^{2}-1}{100^{2}}\left(v_{i}\right)^{2}} \geq 1-\sqrt{1-\frac{1-\rho^{2}}{100^{2}}\left(v_{i}\right)^{2}}, \\
& \sqrt{\rho^{2}-\frac{\rho^{2}-1}{100^{2}}\left(v_{i}\right)^{2}}+\sqrt{1-\frac{1-\rho^{2}}{100^{2}}\left(v_{i}\right)^{2}} \geq 1+\rho .
\end{aligned}
$$

Since both sides are strictly positive, the inequality is preserved under squaring

$$
\sqrt{\rho^{2}-\frac{\rho^{2}-1}{100^{2}}\left(v_{i}\right)^{2}} \sqrt{1-\frac{1-\rho^{2}}{100^{2}}\left(v_{i}\right)^{2}} \geq \rho,
$$

again, using that both sides are positive, we have

$$
\begin{gathered}
\left(\rho^{2}-\frac{\rho^{2}-1}{100^{2}}\left(v_{i}\right)^{2}\right)\left(1-\frac{1-\rho^{2}}{100^{2}}\left(v_{i}\right)^{2}\right) \geq \rho^{2}, \\
\left.-\left(\left(v_{i}\right)^{2}-100^{2}\right)\left(v_{i}\right)^{2}\right) \geq 0,
\end{gathered}
$$

which is equivalent to $v_{i}^{2} \in\left[0,100^{2}\right]$ or $v_{i} \in[0,100]$. Thus, for all realizations of $v_{i}$, the condition $b_{i}^{1} \geq b_{i}^{2}$ is not binding. Since by the standard arguments (analogously to the asymmetric first-price auction), the first-order conditions are necessary and sufficient, bid functions are mutual best responses. Uniqueness: The constructed equilibrium was derived from the necessary conditions and, hence, is unique within the class of symmetric, strictly monotone functions. Bidding zero is not an equilibrium, a bid with "flat parts" is also ruled out by the standard argument that the best response to such a bid necessarily involves gaps and, hence, is not a $C^{1}$ function, the constructed equilibrium is within monotone and symmetric class.

Vickrey auction: Existence: In the Vickrey auction, bidder never affects the prices of units won and, hence, bidding the true values is a weakly dominant strategy, and it constitutes an equilibrium. 